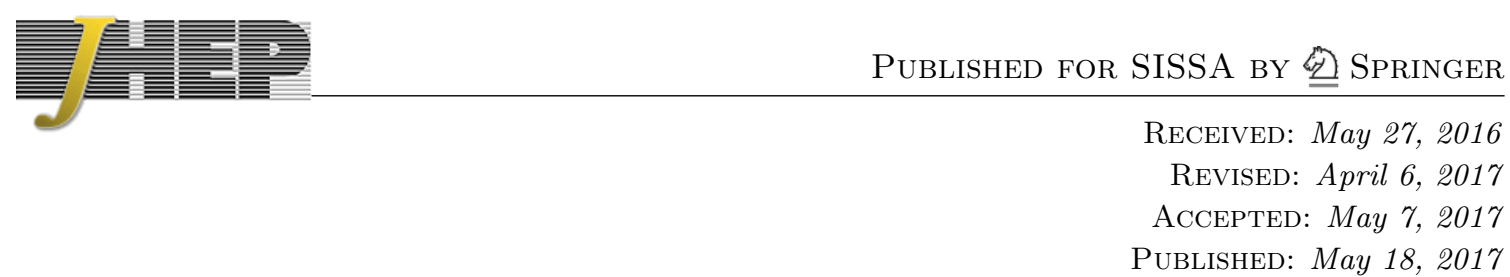

\title{
Bound states of spinning black holes in five dimensions
}

\section{P. Marcos Crichigno, Flavio Porri and Stefan Vandoren}

Institute for Theoretical Physics and Spinoza Institute, Utrecht University, Leuvenlaan 4, 3854 CE Utrecht, The Netherlands

E-mail: p.m.crichigno@uu.nl, f.porri@uu.nl, S.J.G.Vandoren@uu.nl

ABSTRACT: We find and study supergravity BPS bound states of five-dimensional spinning black holes in asymptotically flat spacetime. These solutions follow from multi-string solutions in six-dimensional minimal supergravity and can be uplifted to F-theory or M-theory. We analyze the regularity conditions and work out the example of a bound state of two black holes in detail. The bound state is supported by fluxes through nontrivial topologies exterior to the horizons and KK momentum. Furthermore, we determine the entropy and compare with other macroscopic BPS solutions.

KEYwords: Black Holes, Supergravity Models, Black Holes in String Theory

ArXiv EPrint: 1603.09729 


\section{Contents}

1 Introduction 1

2 Multiple strings in 6d 2

2.1 Supersymmetric solutions in $6 \mathrm{~d}$

$\begin{array}{lll}2.2 & \text { Bubble equations } & 7\end{array}$

2.3 Dualities and charges 8

3 Reduction to five dimensions $\quad 10$

$\begin{array}{ll}3.1 \text { Horizons and conserved charges } & 11\end{array}$

3.2 A comment on notations 13

4 Bound states of two black holes in 5d 14

$\begin{array}{ll}\text { 4.1 Solving the bubble equations } & 15\end{array}$

$\begin{array}{lll}4.2 & \text { Spacetime regularity } & 16\end{array}$

$\begin{array}{lll}4.3 & \text { Global charges } & 18\end{array}$

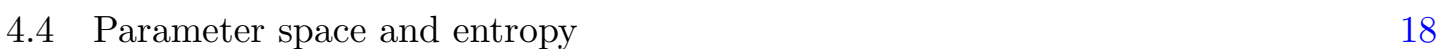

5 Summary and discussion $\quad 21$

A 6d solution and reduction to $5 \mathrm{~d} \quad 22$

A.1 The 1-forms $\chi, \beta, \omega \quad 22$

A.2 Asymptotics 23

A.3 Reduction to 5d 24

B Five-dimensional metrics $\quad 26$

B.1 Asymptotics 26

B.2 Near a center 26

\section{Introduction}

The phase structure of asymptotically flat five-dimensional BPS objects in supergravity is rich and intricate. For instance, there are spinning black holes [1], black rings [2], black hole horizons with Lens space topologies [3], and BPS smooth geometries with no horizon (see [4] and references therein). Moreover, there can be bound states between these objects, such as concentric black rings or black saturns $[5,6]$, or a bound state of a black hole with smooth centers outside the black hole horizon. For a review, see e.g. [7]. In this paper, we investigate the possibility of having bound states of spinning black holes, where each of the black holes has an $S^{3}$ horizon topology.

All these objects can be realized microscopically in string theory. The case with least supersymmetry is M-theory on a Calabi-Yau threefold $X$, or F-theory on $X \times S^{1}$ for elliptic 
$X$. When an F-theory picture is available, a $5 \mathrm{~d}$ black hole arises from a $6 \mathrm{~d}$ black string by wrapping a D3-brane over $\mathcal{C} \times S^{1}$, where $\mathcal{C}$ is a curve in the base of $X$ [8]. Multicenter bound states of spinning black holes arise when $\mathcal{C}$ degenerates into multiple curve components of lower genus [9]. This work served as a motivation for the present study. Since the Ftheory picture describes the black holes as $6 \mathrm{~d}$ black strings wrapped over a circle, we are also led to investigate the question of the existence of multicentered black strings in six dimensions. These multicentered string configurations can also be constructed from type IIB compactifications on $K 3$ or $T^{4}$. Such centers might bind or not in spacetime, and we derive the conditions in $6 \mathrm{~d}$ supergravity for them to form regular BPS bound states. Upon reducing on $S^{1}$, they describe black hole bound states in five dimensions. Our analysis will be done in minimal $(1,0)$ supergravity, which has an F-theory lift in terms of elliptically fibered $X$ with base $\mathbb{P}^{2}$. In $5 \mathrm{~d}$, the bound state black hole system will therefore be a BPS solution of $5 \mathrm{~d}$ supergravity coupled to one vector multiplet. ${ }^{1}$ We expect these bound states to persist in the presence of additional matter multiplets. This would be relevant for string compactifications with more supersymmetry, such as M-theory on $T^{6}$ or IIB on $T^{5}$.

One of the questions we address is whether regular, multicenter solutions exist in regions of parameter space where the single-center solutions would violate the cosmic censorship bound (CCB). This was addressed in the case in which only one center has a finite-size horizon in $[3,12,13]$. However, to the best of our knowledge, a detailed analysis of the case with multiple horizons has not been carried out. Although the local form of the solutions is known, there are various regularity conditions that must be imposed on the metric for the solution to be a good background, leading to a set of nontrivial constraints on the parameters describing the local solution. Whether there is a nonzero space of solutions to these constraints requires a careful analysis.

In this paper, we carry out this analysis in the case of a solution describing two identical finite-size spinning black holes, and a smooth center. The space contains two topological two-cycles connecting the black holes to the smooth center, and the whole system is bound by a nonzero flux through these cycles. We find that there is a narrow region around the $\mathrm{CCB}$ where these configurations exist and are everywhere regular. Moreover, their entropy dominates over the single-centered black hole in a small subregion where both exist.

We begin our analysis with a description of multi-string solutions in six dimensions in section 2. In section 3 we reduce over a circle to five dimensions and in section 4 we discuss the bound states of $5 \mathrm{~d}$ black holes. We end with some discussion in section 5 , and give some technical details in the appendices.

\section{Multiple strings in $6 \mathrm{~d}$}

Consider F-theory on an elliptically fibered Calabi-Yau threefold $X$ with base $B$ [14-16]. In six dimensions this gives rise to the Poincaré multiplet (containg the graviton and a self-dual tensor), $n_{T}=h^{1,1}(B)-1$ tensor multiplets (with anti-selfdual tensors), and

\footnotetext{
${ }^{1}$ Of course, there are also BPS black hole solutions in minimal 5d supergravity [10, 11], but these do not uplift to F-theory. Therefore, there is no underlying microscopic description in terms of a $6 \mathrm{~d}$ black string with an $\mathrm{AdS}_{3}$ near horizon factor whose entropy is governed by a $\mathrm{CFT}_{2}$.
} 
$n_{V}=h^{1,1}(X)-h^{1,1}(B)-1$ vector multiplets. The tensors descend from the RR-four form

$$
C_{(4)}=\sum_{i=1}^{h^{1,1}(B)} C_{(2)}^{i} \wedge \alpha_{i}, \quad C_{(2)}^{i}=\int_{\gamma^{i}} C_{(4)},
$$

where $\alpha_{i}$ and $\gamma^{i}$ form a basis of harmonic $(1,1)$-forms and dual two-cycles respectively. There are also $n_{H}=h^{2,1}(X)$ hypermulitplets from the complex structure deformations of $X$ but they play no role in our analysis and are frozen to constant values. The simplest setup for the study of black holes is when the base is chosen to be $B=\mathbb{P}^{2}$, for which there are no vector multiplets and no tensor multiplets, so we are led to pure minimal chiral $(1,0)$ supergravity in six dimensions, with bosonic fields the metric and a self-dual three-form $\widehat{G}=\mathrm{d} C_{(2)}$. The BPS equations in this supergravity theory were studied and analyzed in [17].

BPS black strings in six dimensions arise from wrapping D3-branes in F-theory over a curve $\mathcal{C}$ in the base $B[8,9,18]$. The near-horizon geometry of such a string in six dimensions is $\mathrm{AdS}_{3} \times S^{3}$. For $B=\mathbb{P}^{2}$, we have only one Kähler class $[H]$ and thus $[\mathcal{C}]=d[H]$ for integer $d$, the degree of the curve. Wrapping the string over an $S^{1}$ with $N$ units of momentum yields a 5 d black hole with entropy [1]

$$
S=2 \pi \sqrt{\frac{d^{2} N}{2}-J^{2}}
$$

where $J$ is the angular momentum of the black hole.

Moving within the class, the curve $\mathcal{C}$ can degenerate into $n_{C}$ curve components and multi-string branches with $d=d_{1}+d_{2}+\ldots+d_{n_{C}}$ can arise [9]. These multiple strings may or may not bind in spacetime; we discuss the conditions under which they form BPS bound states in subsection 2.2 .

\subsection{Supersymmetric solutions in $6 \mathrm{~d}$}

The bosonic content of six-dimensional minimal supergravity is the graviton $\hat{g}_{\hat{\mu} \hat{\nu}}$ and a selfdual three-form field $\widehat{G}_{\hat{\mu} \hat{\nu} \hat{\rho}}$. All supersymmetric solutions of minimal supergravity in $6 \mathrm{~d}$ were described in [17] (for subsequent work see, e.g., [19]), which we closely follow below. The metric is given $b^{2}$

$$
\mathrm{d} s_{6}^{2}=-2 H^{-1}(\mathrm{~d} u+\beta)\left(\mathrm{d} v+\omega-\frac{\mathcal{F}}{2}(\mathrm{~d} u+\beta)\right)+H \mathrm{~d} s_{\mathrm{HK}_{4}}^{2},
$$

where $\mathrm{d} s_{\mathrm{HK}_{4}}^{2}$ is a four-dimensional hyperkähler base and $\beta, \omega$ are 1-forms on $\mathrm{HK}_{4}$. The vector field $\partial_{v}$ is a null Killing vector field. In full generality $\mathcal{F}=\mathcal{F}(u, x), H=H(u, x)$, where $x$ are coordinates in the base, but here we consider the case in which $\partial_{u}$ is a (spacelike) Killing vector, i.e.,

$$
\mathcal{F}=\mathcal{F}(x), \quad H=H(x) .
$$

\footnotetext{
${ }^{2}$ Here we adopt the signature convention mostly plus, unlike [17], and redefined the function $\mathcal{F}_{\text {here }}=$ $-\mathcal{F}_{\text {there }}$.
} 
In this case the 3 -form field is given by

$$
\widehat{G}=\frac{1}{2} *_{4} \mathrm{~d} H-\frac{1}{2} e^{+} \wedge(\mathrm{d} \omega)^{-}+\frac{1}{2} H^{-1} e^{-} \wedge \mathrm{d} \beta-\frac{1}{2} e^{+} \wedge e^{-} \wedge H^{-1} \mathrm{~d} H,
$$

where

$$
e^{+}=H^{-1}(\mathrm{~d} u+\beta), \quad e^{-}=\mathrm{d} v+\omega-\frac{\mathcal{F}}{2}(\mathrm{~d} u+\beta), \quad \mathrm{d} \omega^{-}=\frac{1}{2}\left(\mathrm{~d} \omega-*_{4} \mathrm{~d} \omega\right) .
$$

For these $u$-independent solutions, one may take the $u$-coordinate to be periodic, $u \sim$ $u+\ell$, thus the total F-theory geometry is

$$
\mathbb{R} \times \mathrm{HK}_{4} \times S^{1} \times C Y_{3}
$$

Within this family of solutions, an interesting class is when $\mathrm{HK}_{4}$ is taken to be a multicenter Gibbons-Hawking (GH) space [20], whose metric has the form of a U(1) fibration over flat $\mathbb{R}^{3}$ :

$$
\mathrm{d} s_{\mathrm{HK}_{4}}^{2}=H_{2}^{-1}(\mathrm{~d} \psi+\chi)^{2}+H_{2} \mathrm{~d} s_{\mathbb{R}^{3}}^{2},
$$

with $\mathrm{H}_{2}$ the harmonic function on $\mathbb{R}^{3}$ :

$$
H_{2}=m_{\infty}+\sum_{a} \frac{m_{a}}{\left|\vec{x}-\vec{x}_{a}\right|}, \quad *_{3} \mathrm{~d} \boldsymbol{\chi}=\mathrm{d} H_{2},
$$

where $m_{a}, a=1, \ldots, N$ are integers. The coordinates in $\mathbb{R}^{3}$ are $(r, \theta, \phi)$, where $\theta \in[0, \pi]$ and $\phi \in[0,2 \pi]$ are coordinates on the round $S^{2}$ and $\psi \in[0,4 \pi]$ is the fiber direction.

Before we proceed describing the full solution, let us review some well known facts about the Gibbons-Hawking metrics (2.7) that will be useful later. Close to a center $\vec{x} \rightarrow \vec{x}_{a}$, the metric becomes $\mathbb{R}^{4} / \mathbb{Z}_{\left|m_{a}\right|}$. In particular, this means that $m_{a} \in \mathbb{Z}$ and for $\left|m_{a}\right|=1$ the metric is locally $\mathbb{R}^{4}$. Another important property is that since the $\psi$-fiber shrinks to zero size as one approaches any of the centers with $m_{a} \neq 0$, there is a nontrivial topological 2-cycle between any two centers, spanned by $\psi$ and any curve connecting them. See figure 1. Although there are $\frac{1}{2} N(N-1)$ number of such cycles, there are a total of $N-1$ independent 2-cycles in homology. The asymptotics of these spaces depend on the value of $m_{\infty}$. For $m_{\infty}=0$ the space is known as multi Eguchi-Hanson, with asymptotics $\mathbb{R}^{4} / \mathbb{Z}_{\left|m_{\mathrm{T}}\right|}$, where $m_{\mathrm{T}}=\sum_{a} m_{a}$. For a single center with $m=1$ the metric is simply flat $\mathbb{R}^{4}$.

The multicenter case with $m_{\infty} \neq 0$ is known as multi Taub-NUT, with asymptotics $\mathbb{R}^{3} \times S^{1}$. In this case one may reduce further along this asymptotic $S^{1}$ to four dimensions.

It should be noted that for standard Gibbons-Hawking metrics one requires $\mathrm{H}_{2}>0$ (and hence $m_{a}>0$ ) to ensure the metric is positive definite. However, this condition can be relaxed here as long as the warp factor $H$ in (2.3) compensates for the sign change in $H_{2}$ and the full $6 \mathrm{~d}$ metric has the correct signature. Indeed, we will be particularly interested in configurations where some $m_{a}=-1$. These metrics were used to construct microstates geometries in $5 \mathrm{~d}[21,22]$ and $6 \mathrm{~d}$ [23] and are often referred to as ambipolar Gibbons-Hawking metrics.

We now return to the description of the full solution. Assuming that the Killing vector $\partial_{\psi}$ of the GH base extends to a symmetry of the full space, the complete supergravity 


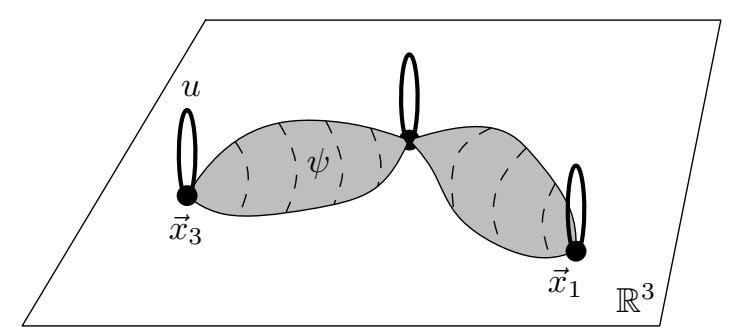

Figure 1. A multi-string configuration in $6 \mathrm{~d}$. The distances between the strings are constrained by the integrability equations (2.22). The base here is a 3-center Gibbons-Hawking space.

background is then given in terms of five additional generic harmonic functions on $\mathbb{R}^{3}$ (see [17] for more details)

$$
\begin{aligned}
H_{1} & =\mu_{\infty}+\sum_{a} \frac{\mu_{a}}{\left|\vec{x}-\vec{x}_{a}\right|}, & H_{3} & =q_{\infty}+\sum_{a} \frac{q_{a}}{\left|\vec{x}-\vec{x}_{a}\right|}, \\
H_{4} & =p_{\infty}+\sum_{a} \frac{p_{a}}{\left|\vec{x}-\vec{x}_{a}\right|}, & H_{5} & =n_{\infty}+\sum_{a} \frac{n_{a}}{\left|\vec{x}-\vec{x}_{a}\right|}, \quad H_{6}=j_{\infty}+\sum_{a} \frac{j_{a}}{\left|\vec{x}-\vec{x}_{a}\right|} .
\end{aligned}
$$

In what follows we adopt a notation in which $r \equiv|\vec{x}|, r_{a} \equiv\left|\vec{x}-\vec{x}_{a}\right|, r_{a b} \equiv\left|\vec{x}_{a}-\vec{x}_{b}\right|$ and denote the set of harmonic functions by

$$
\mathbb{H}=\left(H_{1}, H_{2}, H_{3}, H_{4}, H_{5}, H_{6}\right) .
$$

The functions $\mathcal{F}, H$ appearing in the metric are given in terms of these by

$$
\mathcal{F}=H_{5}+H_{2}^{-1} H_{4}^{2}, \quad H=H_{1}+H_{2}^{-1} H_{3} H_{4},
$$

and the 1-forms $\beta=\beta_{\psi}(\mathrm{d} \psi+\chi)+\boldsymbol{\beta}_{i} \mathrm{~d} x^{i}$ and $\omega=\omega_{\psi}(\mathrm{d} \psi+\chi)+\boldsymbol{\omega}_{i} \mathrm{~d} x^{i}$ are given by

$$
\begin{array}{rlrl}
\beta_{\psi} & =H_{2}^{-1} H_{3}, & *_{3} \mathrm{~d} \boldsymbol{\beta}=-\mathrm{d} H_{3}, \\
\omega_{\psi}=H_{2}^{-2} H_{3} H_{4}^{2}+H_{2}^{-1}\left(H_{1} H_{4}+\frac{1}{2} H_{5} H_{3}\right)+H_{6}, & *_{3} \mathrm{~d} \boldsymbol{\omega}=\langle\mathbb{H}, \mathrm{d} \mathbb{H}\rangle,
\end{array}
$$

where we introduced the symplectic product $\langle u, v\rangle \equiv u^{\top} \Omega v$, with

$$
\Omega=\left(\begin{array}{cccccc}
0 & 0 & 0 & -1 & 0 & 0 \\
0 & 0 & 0 & 0 & 0 & 1 \\
0 & 0 & 0 & 0 & \frac{1}{2} & 0 \\
1 & 0 & 0 & 0 & 0 & 0 \\
0 & 0 & -\frac{1}{2} & 0 & 0 & 0 \\
0 & -1 & 0 & 0 & 0 & 0
\end{array}\right) .
$$

It is convenient to denote the residues and constant parts in $\mathbb{H}$ by the vectors

$$
\Gamma_{a}=\left(\mu_{a}, m_{a}, q_{a}, p_{a}, n_{a}, j_{a}\right), \quad \Gamma_{\infty}=\left(\mu_{\infty}, m_{\infty}, q_{\infty}, p_{\infty}, n_{\infty}, j_{\infty}\right),
$$

The symplectic pairing acts naturally on these by

$$
\left\langle\Gamma_{a}, \Gamma_{b}\right\rangle=p_{a} \mu_{b}-\mu_{a} p_{b}+m_{a} j_{b}-m_{b} j_{a}+\frac{1}{2}\left(q_{a} n_{b}-q_{b} n_{a}\right) .
$$


As shown in [17], the solution reviewed above is the most general $u$-independent solution with a Gibbons-Hawking base space whose Killing vector field $\partial_{\psi}$ extends to a symmetry of the full solution. Specifying a particular local solution in this class amounts to giving a set of locations $\left\{\vec{x}_{a}\right\}$ of the poles in the harmonic functions, their residues $\left\{\Gamma_{a}\right\}$ and the asymptotic values $\Gamma_{\infty}$. To write the solution explicitly one must determine the 1-forms $\boldsymbol{\chi}, \boldsymbol{\beta}, \boldsymbol{\omega}$ from equations (2.8), (2.12) and (2.13), respectively. The expression for these is given in appendix A.1 in a simplified case where all the GH centers lie on a single line inside $\mathbb{R}^{3}$.

The asymptotics of the metric (2.3) is controlled by the asymptotics of the GH base and the behavior of the functions $H, \mathcal{F}$ and 1-forms $\omega, \beta$ as $r \rightarrow \infty$. Setting $m_{\infty}=0$ and requiring that asymptotically $H, \mathcal{F} \rightarrow 1$ and $\omega, \beta \rightarrow 0$, the metric asymptotes to $\mathbb{R}^{1,1} \times \mathbb{R}^{4} / \mathbb{Z}_{m_{\mathrm{T}}}$, where $m_{\mathrm{T}} \equiv \sum_{a} m_{a}$ must be positive for the metric to have the correct signature. See appendix A.2 for details.

Black strings and black tubes. The single black string solution is obtained by taking the harmonic functions (2.8) and (2.9) to have a single pole at the origin with residues $\Gamma=(\mu, m, q, p, n, j)$, with $m$ positive integer ${ }^{3}$ and constant parts $\Gamma_{\infty}$ given by (A.7). The metric (2.3) then reads

$$
\begin{aligned}
\mathrm{d} s^{2}= & -2\left(1+\frac{\widetilde{Q}}{4 \sqrt{2} m r}\right)^{-1}\left[\mathrm{~d} v+\frac{J_{\psi}}{8 m^{2} r}(\mathrm{~d} \psi+m \cos \theta \mathrm{d} \phi)-\frac{1}{2}\left(1+\frac{Q}{4 m r}\right) \mathrm{d} u^{\prime}\right] \mathrm{d} u^{\prime} \\
& +\left(1+\frac{\widetilde{Q}}{4 \sqrt{2} m r}\right)\left[\frac{r}{m}(\mathrm{~d} \psi+m \cos \theta \mathrm{d} \phi)^{2}+m \frac{\mathrm{d} r^{2}}{r}+m r \mathrm{~d} \Omega_{2}^{2}\right]
\end{aligned}
$$

where we defined $u^{\prime}=u+\frac{q}{m} \psi$. Since $u$ and $\psi$ are both periodic, we must impose the quantization condition $\frac{4 \pi}{\ell} \frac{q}{m} \in \mathbb{Z}$ in order for the reparametrization to be globally defined. We also introduced the combinations

$$
\widetilde{Q}=4 \sqrt{2}(\mu m+q p), \quad Q=4\left(n m+p^{2}\right), \quad J_{\psi}=8\left(q p^{2}+\mu p m+\frac{q n}{2} m+j m^{2}\right) .
$$

The spacetime (2.17) is asymptotically $S^{1} \times \mathbb{R}^{1,4} / \mathbb{Z}_{m}$ where the $S^{1}$ is parametrized by $u^{\prime}$ and has an event horizon at $r=0$. The near-horizon geometry ${ }^{4}$ is a direct product of an extremal BTZ black hole and a round $S^{3} / \mathbb{Z}_{m}$. The Bekenstein-Hawking entropy associated to the black string horizon reads

$$
S=\frac{\text { Area }}{4 G_{6}}=2 \pi \sqrt{\frac{Q \widetilde{Q}^{2}}{2 m^{2}}-\frac{J_{\psi}^{2}}{m^{2}}},
$$

where we chose conventions [18] in which $G_{6}=\frac{\ell \pi}{4}$, with $\ell$ the period of $u^{\prime}$. Setting $m=1$ and with the identification with F-theory quantities $J=J_{\psi}, N=Q, d=\widetilde{Q}$, this matches

\footnotetext{
${ }^{3}$ For $m \neq 1$ the spacetime is a $\mathbb{Z}_{m}$ orbifold which can be undone choosing $\psi$ to have period $4 \pi m$. We do not do this here since we want to obtain an entropy formula for a generic set of charges.

${ }^{4} \mathrm{~A}$ solution which is globally $\mathrm{BTZ} \times S^{3}$ can be obtained by taking a single center and setting $\Gamma_{\infty}=0$. For other six-dimensional solutions that asymptote to $\mathrm{AdS}_{3} \times S^{3}$ see e.g. [24].
} 
the entropy (2.2). Since the F-theory quantities are quantized in the microscopic theory, the charges $J_{\psi}, Q, \widetilde{Q}$ are integers, which explains our choice of normalizations in (2.18).

The extension to the multi-string case is straightforward. Taking a generic configurations with charge vectors $\left\{\Gamma_{a}\right\}$ the metric close to any center with $m_{a} \neq 0$ will resemble the $r \rightarrow 0$ limit of (2.17) with coefficients $\widetilde{Q}_{a}, Q_{a}$ and $J_{\psi a}$. In fact, unlike the case of a single string, one may (and we will) allow for some $m_{a}<0$ as long as the asymptotic condition $m_{\mathrm{T}}>0$ is satisfied. The entropy of each string is given by (2.19) with the corresponding charges.

Although not the focus of the present paper, another interesting possibility is when $m_{a}=0$ for some centers. In this case the topology of the horizon at $\vec{x}=\vec{x}_{a}$ degenerates into

$$
S_{u}^{1} \times S^{3} / \mathbb{Z}_{\left|m_{a}\right|} \rightarrow S_{u}^{1} \times S^{1} \times S^{2},
$$

and the object is then a (circular) black tube rather than a black string. This is nothing but the $6 \mathrm{~d}$ uplift of the supersymmetric black ring [2]. Although the entropy formula (2.19) might look singular, it is still valid in the $m_{a}=0$ case by taking the limit (see expressions (2.18)).

We will assume $m_{a} \neq 0$ for the rest of the paper since we are interested in black strings (black holes) in $6 \mathrm{~d}(5 \mathrm{~d})$. Next, we discuss the conditions for these objects to form bound states.

\subsection{Bubble equations}

Although any set of locations $\vec{x}_{a}$ of the strings in $\mathbb{R}^{3}$ provide local supergravity solutions, these will typically have Dirac string-like singularities. In similar settings in four [25] and five dimensions [21], it is well known that imposing the absence of such singularities leads to a constraint on the relative locations of the GH centers. This is also the case in six dimensions (see e.g. [26]). As in the lower-dimensional cases, this arises from requiring the 1-form $\boldsymbol{\omega}$ appearing in the metric to be globally defined, which implies

$$
\mathrm{d}^{2} \boldsymbol{\omega}=0 \text {. }
$$

Taking $\mathrm{d} *_{3}$ on both sides of $(2.13)$ leads to

$$
\sum_{b \neq a} \frac{\left\langle\Gamma_{a}, \Gamma_{b}\right\rangle}{r_{a b}}=\left\langle\Gamma_{\infty}, \Gamma_{a}\right\rangle, \quad a=1, \ldots, N
$$

These equations impose constraints on the relative distances $r_{a b}$ in $\mathbb{R}^{3}$ of the GH centers and their charges. These are usually referred to as "bubble equations," because they control the size of the "bubbles," or 2-cycles, in the Gibbons-Hawking base (see e.g. [7]). In a similar setting in four dimensions [25], they are referred to as "integrability equations."

We note that summing over $a$ on both sides of (2.22), the left-hand side vanishes identically and thus a consistency requirement is

$$
\sum_{a}\left\langle\Gamma_{\infty}, \Gamma_{a}\right\rangle \equiv\left\langle\Gamma_{\infty}, \Gamma_{\mathrm{T}}\right\rangle=0,
$$

which can be interpreted as the condition that there are no Dirac strings running to infinity. 
The constraint (2.22) coincides with the bubble equations found in five dimensions [21]. This is not hard to explain. As we will discuss in section 3, a 5d BPS solution (in the timelike class) is obtained from the $6 \mathrm{~d}$ solution by reducing along the direction $u \sim u+\ell$. Since the bubble equations depend only on the GH base, which is unaffected by the dimensional reduction, these coincide in $5 \mathrm{~d}$ and $6 \mathrm{~d}$.

\subsection{Dualities and charges}

As reviewed above, the class of solutions considered in this paper is characterized by the six harmonic functions $\mathbb{H}=\left(H_{1}, H_{2}, H_{3}, H_{4}, H_{5}, H_{6}\right)$. Since these are generic harmonic functions, and a linear combination of harmonic functions is harmonic, it is clear that sending $\mathbb{H} \rightarrow g \mathbb{H}$ with $g \in \mathrm{GL}(6, \mathbb{R})$ will send a solution to a solution. An interesting question is whether this operation preserves regularity, in particular the absence of Diracstring singularities. One way to ensure this is if the bubble equations (2.22) are preserved, which leads us to consider the subgroup, $\operatorname{Sp}(6, \mathbb{R})$, preserving the symplectic product, i.e., $g^{\top} \Omega g=\Omega$.

One example of such transformations is given by the two-parameter set of transformations

$$
g_{\text {gauge }}=\left(\begin{array}{cccccc}
1 & -g_{1} g_{2} & -g_{1} & -g_{2} & 0 & 0 \\
0 & 1 & 0 & 0 & 0 & 0 \\
0 & g_{2} & 1 & 0 & 0 & 0 \\
0 & g_{1} & 0 & 1 & 0 & 0 \\
0 & -g_{1}^{2} & 0 & -2 g_{1} & 1 & 0 \\
-g_{1} & \frac{1}{2} g_{2} g_{1}^{2} & \frac{1}{2} g_{1}^{2} & g_{1} g_{2} & -\frac{1}{2} g_{2} & 1
\end{array}\right) \text {, }
$$

where $g_{1,2}$ are real parameters. In fact, these transformations form a two-dimensional subgroup of $\operatorname{Sp}(6, \mathbb{R})$ and it is easy to see that they leave the functions $H, \mathcal{F}$, as well as the 1 -form $\omega$ invariant. The 1 -form $\beta$ transforms by an exact term: $\beta \rightarrow \beta-g_{2} \mathrm{~d} \psi$, which can be undone by the coordinate transformation $u \rightarrow u+g_{2} \psi$. Furthermore, since the function $\mathrm{H}_{2}$ is invariant, the effect of the transformation (2.24) is a simple, unphysical, change of coordinates. The explicit action on the residues reads

$$
\begin{aligned}
\mu_{a} & \rightarrow \mu_{a}-g_{1} q_{a}-g_{2} p_{a}-g_{1} g_{2} m_{a}, \quad m_{a} \rightarrow m_{a}, \quad q_{a} \rightarrow q_{a}+g_{2} m_{a}, \\
p_{a} & \rightarrow p_{a}+g_{1} m_{a}, \quad n_{a} \rightarrow n_{a}-2 g_{1} p_{a}-g_{1}^{2} m_{a}, \\
j_{a} & \rightarrow j_{a}-g_{1} \mu_{a}-\frac{1}{2} g_{2} n_{a}+g_{1} g_{2} p_{a}+\frac{1}{2} g_{1}^{2} q_{a}+\frac{1}{2} g_{2} g_{1}^{2} m_{a} .
\end{aligned}
$$

In particular, we note that one may always set the $p_{a}, q_{a}$ charges of one center to zero by choosing $g_{1}, g_{2}$ appropriately (provided $m_{a} \neq 0$ ). In the context of M-theory on $T^{6}$ these are referred to as "gauge" transformations [27]. This symmetry can be used to construct physically relevant combinations of the residues, as we discuss next.

For an $N$ number of centers, there are a total of $6 N$ residues in the harmonic functions. ${ }^{5}$ Due to the redundancy we just described, the residues themselves are not physical

\footnotetext{
${ }^{5}$ In principle there are six more parameters in $\Gamma_{\infty}$ which should be considered in the counting. In fact, one can construct gauge-invariant combinations analogous to (2.26). However, the latter vanish in the asymptotically flat solutions considered here.
} 
quantities. Since the redundancy is characterized by two parameters $g_{1,2}$ there should be a total of $6 N-2$ gauge-invariant combinations. These are the $m_{a}$ 's themselves, together with the $5 N-2$ independent combinations

$$
\begin{aligned}
& \widetilde{Q}_{a} \equiv 4 \sqrt{2}\left(\mu_{a} m_{a}+q_{a} p_{a}\right), \quad Q_{a} \equiv 4\left(n_{a} m_{a}+p_{a}^{2}\right), \\
& J_{a}^{\psi} \equiv 8\left(q_{a} p_{a}^{2}+\mu_{a} p_{a} m_{a}+\frac{q_{a} n_{a}}{2} m_{a}+j_{a} m_{a}^{2}\right), \\
& f_{a, a+1} \equiv \frac{q_{a+1}}{m_{a+1}}-\frac{q_{a}}{m_{a}}, \quad \widetilde{f}_{a, a+1} \equiv \sqrt{2}\left(\frac{p_{a+1}}{m_{a+1}}-\frac{p_{a}}{m_{a}}\right) .
\end{aligned}
$$

In the last line we have assumed $m_{a} \neq 0$ for all $a$. The quantities above have a clear physical interpretation, as we will discuss in section 3. In the case of a single GH center, these quantities reduce exactly to the quantities (2.18).

Another interesting subgroup of $\operatorname{Sp}(6, \mathbb{R})$ is given by

$$
g_{\mathrm{SF}}=\left(\begin{array}{cccccc}
1 & 0 & 0 & 0 & 0 & -2 \gamma_{1} \\
-2 \gamma_{1} \gamma_{2} & 1 & \gamma_{2} & 2 \gamma_{1} & -\gamma_{1}^{2} & 2 \gamma_{1}^{2} \gamma_{2} \\
-2 \gamma_{1} & 0 & 1 & 0 & 0 & 2 \gamma_{1}^{2} \\
-\gamma_{2} & 0 & 0 & 1 & -\gamma_{1} & 2 \gamma_{1} \gamma_{2} \\
0 & 0 & 0 & 0 & 1 & -2 \gamma_{2} \\
0 & 0 & 0 & 0 & 0 & 1
\end{array}\right)
$$

where $\gamma_{1,2}$ are real parameters. These transformations do not leave the quantities (2.26) invariant. Instead, they lead to a new solution, characterized by the transformed quantities $\left\{m_{a}^{\prime}, Q_{a}^{\prime}, \widetilde{Q}_{a}^{\prime}, J_{a}^{\psi^{\prime}}, f_{a, a+1}^{\prime}, \widetilde{f}_{a, a+1}^{\prime}\right\}$. Although the transformation acts non-trivially on these quantities one can see that the entropy (2.19) is invariant. Since the transformation preserves the bubble equations, the new supergravity background will necessarily be free of Dirac singularities, provided the original background is. In the case of M-theory on $T^{6}$, these correspond to a subgroup of the $E_{7(7)}$ U-duality group, referred to as generalized spectral flow transformations in [26].

Finally, another subgroup of $\operatorname{Sp}(6, \mathbb{R})$ leaving the entropy invariant is given by

$$
g_{\text {resc. }}=\left(\begin{array}{cccccc}
\beta_{1} & 0 & 0 & 0 & 0 & 0 \\
0 & \beta_{2} & 0 & 0 & 0 & 0 \\
0 & 0 & \beta_{1}^{2} \beta_{2} & 0 & 0 & 0 \\
0 & 0 & 0 & \beta_{1}^{-1} & 0 & 0 \\
0 & 0 & 0 & 0 & \beta_{1}^{-2} \beta_{2}^{-1} & 0 \\
0 & 0 & 0 & 0 & 0 & \beta_{2}^{-1}
\end{array}\right),
$$

with $\beta_{1,2}$ real parameters, which acts by a simple rescaling of the residues.

Before we proceed we make a brief comment. We have shown that the particular $\mathrm{Sp}(6, \mathbb{R})$ group elements $(2.24),(2.27)$ and $(2.28)$ leave the entropy invariant. In fact it is not difficult to show that combinations of these transformations form the most general subgroup $H \subset \operatorname{Sp}(6, \mathbb{R})$ with this property and that they form the direct product

$$
H=\mathrm{SL}(2, \mathbb{R}) \times \mathrm{SL}(2, \mathbb{R}),
$$

where each factor is a combination of all three types of transformations described above. The meaning of the $\operatorname{Sp}(6, \mathbb{R})$ group and its consequences will be studied in [28]. 


\section{Reduction to five dimensions}

Compactification of F-theory on $X \times S^{1}$ yields an effectively five-dimensional theory which is the circle reduction of the $6 \mathrm{~d}$ theory we considered in the previous section. By wrapping the black strings over $S_{u}^{1}$ with quantized momenta, we obtain charged spinning black holes in five dimensions. In this section, we discuss the dimensional reduction from $6 \mathrm{~d}$ to $5 \mathrm{~d}$.

Dimensional reduction of $6 \mathrm{~d}$ minimal supergravity to $5 \mathrm{~d}$ leads to the following bosonic fields: a metric $g_{\mu \nu}$, a scalar $\varphi$, and two vector fields $A_{\mu}$ and $\widetilde{A}_{\mu}$. For further details we refer the reader to appendix A.3. Reduction of the $6 \mathrm{~d}$ metric and three-form along the $u$-direction ${ }^{6}$ yields

$$
\begin{aligned}
\mathrm{d} s_{6}^{2} & =\mathrm{e}^{2 \varphi}(\mathrm{d} u+A)^{2}+\mathrm{e}^{-2 \varphi / 3} \mathrm{~d} s_{5}^{2}, \\
\widehat{G} & =G+\frac{1}{2} \mathrm{~d} \widetilde{A} \wedge(\mathrm{d} u+A),
\end{aligned}
$$

with the five-dimensional, Einstein-frame metric

$$
\mathrm{d} s_{5}^{2}=-f^{2}(\mathrm{~d} t+\omega)^{2}+f^{-1} \mathrm{~d} s_{\mathrm{HK}_{4}}^{2}, \quad f^{-1}=\left(H^{2} \mathcal{F}\right)^{1 / 3},
$$

where we relabeled $\mathrm{d} t=\mathrm{d} v$. For BPS solutions of the Gibbons-Hawking type, the radius and the two vector fields read

$$
\begin{aligned}
e^{2 \varphi} & =H^{-1} \mathcal{F}, \\
A & =-\left(H_{5}+H_{2}^{-1} H_{4}^{2}\right)^{-1}(\mathrm{~d} t+\omega)+\beta, \\
\widetilde{A} & =-\left(H_{1}+H_{2}^{-1} H_{3} H_{4}\right)^{-1}(\mathrm{~d} t+\omega)+\gamma,
\end{aligned}
$$

where $\beta$ satisfies (2.12) and $\gamma=\gamma_{\psi}(\mathrm{d} \psi+\chi)+\gamma_{i} \mathrm{~d} x^{i}$ similarly satisfies

$$
\gamma_{\psi}=H_{2}^{-1} H_{4}, \quad *_{3} \mathrm{~d} \gamma=-\mathrm{d} H_{4} .
$$

These solutions - and their extensions with an arbitrary number of vector multiplets were studied in [6].

Note that the five-dimensional solution is still described by six harmonic functions and the residues and locations of the centers are still constrained by the same bubble equations (2.22). One then concludes that the five-dimensional solution describes a bound state of black holes if and only if the six-dimensional solution corresponds to a bound state of black strings. Before discussing the variety of possible five-dimensional configurations we briefly comment on the M-theory setup of these solutions.

The five-dimensional theory in question can also be obtained directly from eleven dimensions by using F-theory/M-theory duality. F-theory on $X \times S^{1}$ is dual to M-theory on $X$. The D3-brane wrapping $\mathcal{C} \times S^{1}$ with $n$ units of momentum is dual to an M2 brane wrapping a curve in the class $n\left[T^{2}\right]+[\mathcal{C}]$, where $\mathcal{C}$ is the curve in the base $B \subset X$, and $T^{2}$ is the elliptic fiber of $X$. In a type IIA setting, this is a bound state of $n$ D2-branes wrapping $T^{2}$ and one D2 wrapping $\mathcal{C}$.

\footnotetext{
${ }^{6}$ Another possibility would be to reduce along the GH fiber $\mathrm{d} \psi$, in this way one can obtain 5 d solutions of the "null" class [11]. We do not discuss this here.
} 


\begin{tabular}{|c|c|c|c|}
\hline KK-monopole & M5-flux & M2-charge & $\partial_{\psi}$ \\
\hline$m_{a}$ & $f_{a, a+1}, \widetilde{f}_{a, a+1}$ & $Q_{a}, \widetilde{Q}_{a}$ & $J_{a}^{\psi}$ \\
\hline
\end{tabular}

Table 1. Relation between residues in the functions $\mathbb{H}$ and charges of M-theory objects.

Generically, M-theory on a Calabi-Yau manifold $X$ gives $n_{V}=h^{1,1}(X)-1$ vector multiplets and $n_{H}=h^{1,2}(X)+1$ hypermultiplets $[29,30] . h^{1,1}(X)$ vectors arise from expanding the eleven dimensional three-form in $H^{2}(X, \mathbb{R})$,

$$
C_{(3)}=\sum_{A=1}^{h^{1,1}(X)} A^{A} \wedge \omega_{A}+\ldots
$$

where the ellipsis denote the terms leading to five-dimensional hypermultiplet scalars. One of these vector fields becomes the graviphoton while the others sit in the $n_{V}$ vector multiplets. The $5 \mathrm{~d}$ real scalars in the vector multiplet correspond to the $h^{1,1}$ (uncomplexified) Kähler moduli of $X$. One combination, however, forms the volume modulus and sits in a hypermultiplet. These hypermultiplets play no role in our analysis, and are frozen to constant values. In the example of the elliptically fibered $X$ over base $B=\mathbb{P}^{2}$, we obtain $5 \mathrm{~d}$ supergravity coupled to a single vector multiplet. The two one-forms from (3.5) correspond to $A$ and $\widetilde{A}$ in (3.1). They define the M2-brane charges, and their duals can support M5-brane flux. The radius field in (3.1) becomes the real scalar in the five-dimensional vector multiplet and measures the inverse area of the elliptic fiber (in 11d Planck units). For black hole solutions, the scalar is subject to the $5 \mathrm{~d}$ attractor mechanism [31, 32], and we find at the horizon

$$
\left.\mathrm{e}^{2 \varphi}\right|_{\text {Hor. }}=\sqrt{2} \frac{Q}{\widetilde{Q}}
$$

At infinity we have set $\mathrm{e}^{2 \varphi} \rightarrow 1$. (See footnote 11.) As an additional remark, we notice that we can truncate the $5 \mathrm{~d}$ theory down to minimal supergravity. This can be done by choosing $\mathcal{F}=H$ such that the radius is constant everywhere, $e^{2 \varphi}=1$. This can be achieved on BPS solutions by taking $H_{1}=H_{5}$ and $H_{3}=H_{4}$, such that, for single centers, $\mu=n$ and $q=p$ and hence $\widetilde{Q}=\sqrt{2} Q$, consistent with (3.6). Bound state solutions we discuss below can therefore be truncated to minimal supergravity as well. Minimal supergravity can be embedded in F-theory by adding and freezing another 5d vector multiplet to the theory. If such a multiplet is not part of the spectrum, an F-theory embedding is not possible, as mentioned in footnote 1 . The M-theory picture does remain though.

The M-theory interpretation of the quantities (2.26) is given in table 1. Thus, the configurations of interest arise from configurations of KK-monopoles and antimonopoles, M2-branes and M5-brane charge fluxes.

\subsection{Horizons and conserved charges}

The dimensional reduction presented above allows one to obtain the full spectrum of fivedimensional BPS solutions in the time-like class [11]: reducing the solution (2.17) along $u$ one obtains, depending on the value of $m$, the BMPV black hole [1] or the black hole with 
lens topology (black lens) recently discussed in [3]; the supersymmetric black ring [2] can be obtained reducing a black tube solution. Finally there can be bound states of all these objects such as concentric black rings $[5,6]$.

The nature of each object, characterized by the topology of the horizon $\mathcal{H}$, in a multicenter solution depends on the choice of charge vectors $\left\{\Gamma_{a}\right\}$ or, rather, the value of the gauge-invariant combinations (2.26). Schematically, ${ }^{7}$ the situation is summarized as follows:

i. Black hole (or black lens)

$$
Q_{a} \widetilde{Q}_{a} \neq 0, m_{a} \neq 0 \quad \Longrightarrow \quad \mathcal{H}_{a} \sim S^{3} / \mathbb{Z}_{\left|m_{a}\right|}
$$

ii. Black ring

$$
Q_{a} \widetilde{Q}_{a} \neq 0, m_{a}=0 \quad \Longrightarrow \quad \mathcal{H}_{a} \sim S^{1} \times S^{2}
$$

iii. Smooth center

$$
Q_{a}=\widetilde{Q}_{a}=J_{\psi a}=0, m_{a} \neq 0 \quad \Longrightarrow \quad \text { no horizon }
$$

The last case can be thought of a limit of the first case; the black hole horizon shrinks to zero size but it does so smoothly and the metric near such a GH center becomes $\mathbb{R}^{4} / \mathbb{Z}_{\left|m_{a}\right|}$. Here we focus our attention on configurations of bound states of black holes with finite $S^{3}$ horizons and, possibly, smooth centers in asymptotically flat $\mathbb{R}^{1,4}$. Rather surprisingly, these solutions have not been studied in the literature in detail. For a study of black hole bound states in asymptotically $\mathrm{AdS}_{3} \times S^{2}$ see [33]. For the rest of the paper, unless otherwise specified, we will assume $m_{a} \neq 0$. Although conical singularities are harmless in string theory, we take $\left|m_{a}\right|=1$ to have black holes with smooth $S^{3}$ horizons. Since asymptotic flatness requires $m_{\mathrm{T}}=1$, we are necessarily led to consider ambipolar GH bases.

In all the cases listed above, the entropy of the corresponding object is given by

$$
S_{a}=\frac{\operatorname{Area}\left(\mathcal{H}_{a}\right)}{4 G_{5}}=\frac{2 \pi}{\left|m_{a}\right|} \sqrt{\frac{Q_{a} \widetilde{Q}_{a}^{2}}{2}-J_{\psi a}^{2}},
$$

where $G_{5}=\pi / 4$ in our conventions.

The charges $Q_{a}, \widetilde{Q}_{a}$ and $J_{\psi a}$ in (2.26) can be given a geometric meaning, as the following integrals performed on the horizons $\mathcal{H}_{a}:{ }^{8}$

$$
\begin{aligned}
J_{\psi a} & =\frac{1}{4 \pi^{2}} \int_{\mathcal{H}_{a}} *_{5} \mathrm{~d} K^{(\psi)}, \\
\widetilde{Q}_{a} & =-\frac{\sqrt{2}}{8 \pi^{2}} \int_{\mathcal{H}_{a}} *_{5} \widetilde{F}, \\
Q_{a} & =-\frac{1}{8 \pi^{2}} \int_{\mathcal{H}_{a}} *_{5} F,
\end{aligned}
$$

\footnotetext{
${ }^{7}$ See appendix B.2 for a more detailed discussion.

${ }^{8}$ The prefactors in the definitions of $Q$ and $\widetilde{Q}$ are due to non-canonical kinetic terms for the gauge fields.
} 
where $K^{(\psi)}$ is the one-form associated to the Killing vector $\partial_{\psi}$. Thus, $Q_{a}, \widetilde{Q}_{a}$ and $J_{\psi a}$ are identified with the two electric charges and the angular momentum, respectively, of the black hole located at $\vec{x}=\vec{x}_{a}$. To interpret the quantities $f_{a, a+1}$ and $\widetilde{f}_{a, a+1}$ we recall the discussion below (2.8). In particular, the fact that a multicenter GH space contains $N-1$ independent 2-cycles, a basis for which is provided by the elements $\mathcal{C}_{a, a+1}$ connecting the centers $x_{a}$ and $x_{a+1}$. Following [7] one can compute the fluxes of the magnetic part of the U(1) field strengths $F$ and $\widetilde{F}$, giving

$$
f_{a, a+1}=\frac{1}{4 \pi} \int_{\mathcal{C}_{a, a+1}} F, \quad \widetilde{f}_{a, a+1}=\frac{\sqrt{2}}{4 \pi} \int_{\mathcal{C}_{a, a+1}} \widetilde{F} .
$$

This also shows that all the combinations (2.26) can be expressed as integrals of gaugeinvariant quantities.

In addition to the individual charges above, for asymptotically flat spacetimes one can define the total charges measured at infinity, given by the Komar integrals

$$
\begin{aligned}
J_{\psi} & =\frac{1}{4 \pi^{2}} \int_{S_{\infty}^{3}} *_{5} \mathrm{~d} K^{(\psi)}=8\left(j_{\mathrm{T}}+\mu_{\mathrm{T}} p_{\mathrm{T}}+\frac{1}{2} n_{\mathrm{T}} q_{\mathrm{T}}+q_{\mathrm{T}} p_{\mathrm{T}}^{2}\right), \\
\widetilde{Q} & =-\frac{\sqrt{2}}{8 \pi^{2}} \int_{S_{\infty}^{3}} *_{5} \mathrm{~d} \widetilde{A}=4 \sqrt{2}\left(\mu_{\mathrm{T}}+p_{\mathrm{T}} q_{\mathrm{T}}\right), \\
Q & =-\frac{1}{8 \pi^{2}} \int_{S_{\infty}^{3}} *_{5} \mathrm{~d} A=4\left(n_{\mathrm{T}}+p_{\mathrm{T}}^{2}\right),
\end{aligned}
$$

where $x_{\mathrm{T}} \equiv \sum_{a} x_{a}$ and we used $m_{\mathrm{T}}=1$ for asymptotically flat spacetimes. We note the the asymptotic charges are not simply given by the sum of the corresponding charges of each center. One can also define the total energy of the solution as the charge associated to the canonical time-like Killing vector $\partial_{t}$ :

$$
M=-\frac{3}{8 \pi^{2}} \int_{S_{\infty}^{3}} *_{5} \mathrm{~d} K^{(t)}=Q+\sqrt{2} \widetilde{Q},
$$

where the second equality is a consequence of the BPS condition.

In the next section we will specialize the setting to multicenter configurations where all the centers lie on a single line, which we take to be the $z$-axis. As a consequence, the solution has an additional $\mathrm{U}(1)$ isometry generated by rotations along the coordinate $\phi$. The corresponding angular momentum is given by

$$
J_{\phi}=\frac{1}{32 \pi^{2}} \int_{S_{\infty}^{3}} *_{5} \mathrm{~d} K^{(\phi)}=\sum_{a} z_{a}\left\langle\Gamma_{a}, \Gamma_{\infty}\right\rangle
$$

where $z_{a}$ is the position of the $a$ th center on the $z$-axis. For these axisymmetric solutions, the equations of motion (2.8), (2.12) and (2.13) can be easily solved for any number of centers. The expressions for the 1 -forms $\boldsymbol{\chi}, \boldsymbol{\beta}, \boldsymbol{\omega}$ in this case are given in appendix A.1.

\subsection{A comment on notations}

The five-dimensional solutions presented in this section can also be viewed as particular instances of the three-charge solutions, considered e.g. in [7], where two of the three vectors multiplets are identified. 
The harmonic functions in [7] are labeled using the $V, K, L, M$ coding which is more standard in the literature about $5 \mathrm{~d}$ solutions. In order to make contact with the notation there, one should identify

$$
M=H_{6}, \quad V=H_{2}, \quad L_{1}=L_{2}=H_{1}, \quad L_{3}=H_{5}, \quad K^{1}=K^{2}=H_{4}, \quad K^{3}=H_{3} .
$$

The three charges of the solution are then identified as

$$
Q_{1}=Q_{2}=\frac{\widetilde{Q}}{\sqrt{2}}, \quad Q_{3}=Q
$$

and (3.11) is consistent with the usual BPS relation between the ADM mass and the charges in the three-charge system:

$$
M=Q_{1}+Q_{2}+Q_{3}
$$

Although the two theories are equivalent at tree-level they are obtained as the low energy limit of two distinct microscopic theories. The one presented here comes from F-theory on $X \sim T^{2} \times \mathbb{P}^{2}$ and the one in [7] from M-theory on $T^{6}$. The quantization conditions on the charges are different in the two cases. The charge $\widetilde{Q}$ is more appropriate for the F-theory compactification [18] and will be used in this paper.

\section{Bound states of two black holes in $5 \mathrm{~d}$}

In this section, we study the simplest configuration of bound black holes with $S^{3}$ horizons in asymptotically flat $\mathbb{R}^{1,4}$ space. The configuration consists of two identical BMPV black holes and a smooth center, bound together by M5-charge flux through nontrivial topological cycles exterior to the horizons. We shall show that, for fixed asymptotic charges, there is a small but finite region in parameter space where the twin black hole solutions exists and is regular, and even a region where it is entropically favored over the single-center black hole with the same asymptotic charges.

Consider a three-center configuration with charge vectors $\Gamma_{a}, a=1,2,3$. Since we are interested in smooth horizons with $S^{3}$ topologies we take $m_{a}= \pm 1$ and since asymptotic flatness requires $m_{\mathrm{T}}=1$, the only choice is $m_{a}=(1,-1,1)$, up to trivial permutations. We take one of these centers, which we choose to be $\Gamma_{2}$, to be smooth, i.e.,

$$
\widetilde{Q}_{2}=Q_{2}=J_{2}=0 \text {. }
$$

Thus, the charge vectors read

$$
\Gamma_{1}=\left(\mu_{1}, 1,0,0, n_{1}, j_{1}\right), \quad \Gamma_{2}=\left(q p,-1, q, p, p^{2}, \frac{1}{2} q p^{2}\right), \quad \Gamma_{3}=\left(\mu_{3}, 1, q_{3}, p_{3}, n_{3}, j_{3}\right),
$$

where we have set $p_{1}=q_{1}=0$ without loss of generality by the gauge transformation (2.25). If $\Gamma_{3}$ was chosen to be a smooth center, this would describe a single black hole and two smooth centers studied in [13]. Here, instead, we set $q_{3}=p_{3}=0$ and assume $Q_{1,3}, \widetilde{Q}_{1,3} \neq 0$, in which case the system corresponds to two BMPV black holes and a smooth center, as shown in figure 2. 


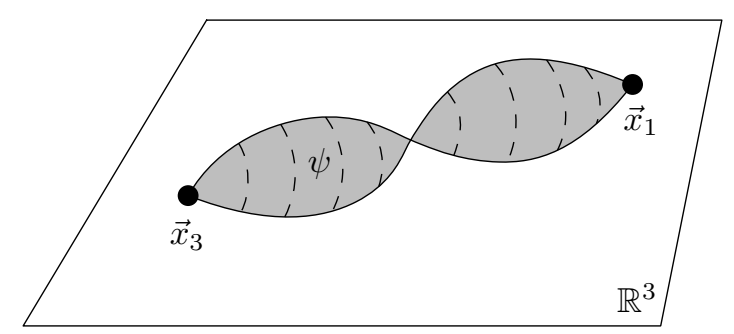

Figure 2. A configuration of two black holes and a smooth center in the middle, bound by flux through the two 2-cycles of the GH base.

Explicitly, the harmonic functions are given by

$$
\begin{array}{lll}
H_{1}=1+\frac{q p}{r}+\frac{\mu_{1}}{r_{1}}+\frac{\mu_{3}}{r_{3}}, & H_{2}=-\frac{1}{r}+\frac{1}{r_{1}}+\frac{1}{r_{3}}, \\
H_{3}=\frac{q}{r}, & H_{4}=\frac{p}{r}, \\
H_{5}=1+\frac{p^{2}}{r}+\frac{n_{1}}{r_{1}}+\frac{n_{3}}{r_{3}}, & H_{6}=j_{\infty}+\frac{q p^{2}}{2 r}+\frac{j_{1}}{r_{1}}+\frac{j_{3}}{r_{3}},
\end{array}
$$

where we have set $\vec{x}_{2}=0$ without loss of generality and $j_{\infty}=-p-\frac{q}{2}$ to ensure $\mathbb{R}^{1,4}$ asymptotics (see (A.7)).

We emphasize that although the space is asymptotically $\mathbb{R}^{1,4}$ the GH base space is nontrivial so this is not a configuration of two BMPV black holes in $\mathbb{R}^{1,4}$. Instead, the geometry contains two nontrivial topological 2-cycles connecting each black hole to the smooth center. As we discuss next, magnetic (or M5-charge) fluxes through these cycles keep the system bound.

\subsection{Solving the bubble equations}

We now study the constraints on the relative locations of the GH centers imposed by the bubble equations. For simplicity, we focus on axisymmetric configurations, where the three GH centers lie on a straight line inside $\mathbb{R}^{3}$, which we take to be the $z$-axis and place the smooth center at the origin. We denote the locations of the two black holes by $\vec{x}_{1}=\left\{0,0, z_{1}\right\}, \vec{x}_{3}=\left\{0,0, z_{3}\right\}$ and choose the orientation of the axis such that $z_{1}>0$.

The bubble equations (2.22) read:

$$
\begin{aligned}
& \frac{2 \mu_{1} p+n_{1} q-p^{2} q-2 j_{1}}{2 z_{1}}+\frac{j_{1}-j_{3}}{\left|z_{1}-z_{3}\right|}=j_{\infty} \\
& \frac{2 \mu_{3} p+n_{3} q-p^{2} q-2 j_{3}}{2\left|z_{3}\right|}+\frac{j_{3}-j_{1}}{\left|z_{1}-z_{3}\right|}=j_{\infty}
\end{aligned}
$$

together with $j_{\infty}+p+\frac{q}{2}=0$, from (2.23).

Let us assume for the moment that $j_{\infty} \neq 0$. We first note that in the case of identical twin black holes, i.e., $\Gamma_{1}=\Gamma_{3} \equiv(\mu, 1,0,0, n, j)$, it follows from (4.4) that $z_{3}= \pm z_{1}$. Thus, to avoid Dirac-string singularities the two black holes must be either symmetrically located with respect to the origin, or sit on top of each other. In the latter case, the configuration is 
in fact a two-center solution, consisting of a single black hole with horizon topology $S^{3} / \mathbb{Z}_{2}$ and a smooth center, studied in [3]. Here, instead, we consider the former case, for which

$$
a \equiv z_{1}=-z_{3}=\frac{2 j-n q+p^{2} q-2 p \mu}{q+2 p} .
$$

Thus, in this simple three-center configuration the distances between the black holes and the smooth center are completely fixed. Since $z_{1}>0$, for consistency we must require

$$
\frac{2 j-n q+p^{2} q-2 p \mu}{q+2 p}>0 \text {. }
$$

This is the first restriction on the parameter space $(\mu, q, p, n, j)$ characterizing the solution.

\subsection{Spacetime regularity}

In order for the solution to be physically acceptable, one must ensure the absence of closed timelike curves (CTCs) anywhere in spacetime [35]. The absence of Dirac-string singularities discussed above is a necessary condition for the absence of CTCs close to the centers [21]. However, as pointed out in [7], this is not enough. For example, in order to avoid CTCs one must also impose

$$
\begin{aligned}
g_{r r} & =H_{2} f^{-1}>0, \\
g_{\psi \psi} & =\frac{1}{f H_{2}}-f^{2} \omega_{\psi}^{2}>0 .
\end{aligned}
$$

A somewhat stronger requirement $[22,36]$, which is sufficient to ensure the absence of CTCs globally, is to demand the metric to be stably causal and that $t$ provides a global time function. This is achieved if and only if

$$
g^{t t}=-f^{-2}+f H_{2} \omega_{\psi}^{2}+f H_{2}^{-1}\left|\boldsymbol{\omega}_{i}\right|^{2}<0
$$

Note that this condition does not necessarily imply both equations in (4.7). If, however, $g_{r r}>0$ is satisfied then (4.8) does imply $g_{\psi \psi}>0$.

We will thus study the constraints imposed by (4.7a) and (4.8) on the parameters characterizing the configuration. We will show that the parameter space where these are satisfied is nonempty and, furthermore, that there is a region where it coexists with the single-center BMPV black hole with the same asymptotic charges and a region where the single-center solution would violate the CCB, but the multicenter is regular. We carry out the analysis for the case of twin black holes, located symmetrically from the smooth center at the distance (4.5) as in figure 2. We begin by analyzing (4.7) close to the centers.

Close to the centers. As discussed in appendix B.2, close to the black hole horizons the regularity conditions $g_{r r}>0$ and $g^{t t}<0$ imply, respectively,

$$
\mu, n>0, \quad-\mu \sqrt{n}<j<\mu \sqrt{n} .
$$


Note that since close to a center $\boldsymbol{\omega}=0$ (see appendix B.2) the condition (4.8) coincides with (4.7b). These are the standard regularity conditions for a single-center BMPV black hole. At the smooth centers the condition $g_{r r}>0$ reads

$$
p^{2}>n+\frac{1}{2} a, \quad p q>\mu+\frac{1}{2} a,
$$

where we have used the fact that $z_{1}=-z_{3} \equiv a$ from (4.5) to simplify the expressions. In addition, using the bubble equation (4.5) one can see that close to the origin $-g^{t t} g_{r r}^{-2}=r^{2}+$ $\mathcal{O}\left(r^{3}\right)$. Thus, if (4.10) holds, $g^{t t}<0$ is automatically satisfied close to the smooth center.

Away from the centers. We begin with the condition $g_{r r}>0$, which amounts to studying the positivity (4.7a) for all $r, \theta$. Remarkably, it is possible to prove that the positivity of this function close to the centers and at infinity is sufficient to ensure this globally. Precisely, under the assumption that the space is asymptotic to $\mathbb{R}^{1,4}$ and using the first inequalities in (4.9) and (4.10) one can prove that $H_{2} f^{-1}>0$ for any $r, \theta$. Thus,

$$
g_{r r}>0 \text { at all centers } \quad \Leftrightarrow \quad g_{r r}>0 \text { everywhere. }
$$

We turn now to the study of possible CTCs, which amounts to studying (4.8) for all $r, \theta$. This is a rather nontrivial constraint and one should not expect the regularity conditions close to the centers (4.9), (4.10) to be sufficient to ensure the absence of CTCs globally. In fact, there are known examples where this is not the case (see e.g. discussion in [7]). However, within this class of solutions we have checked numerically in a vast number of instances of solutions to (4.9), (4.10), that $g^{t t}<0$ is, in fact, satisfied on the entire coordinate patch. Thus, the region in the five-dimensional parameter space $(\mu, q, p, n, j)$ where the configuration of twin back holes is globally regular (within our numerical analysis) is given by (4.6), (4.9) and (4.10). Before we analyze this parameter space in more detail, we discuss the physical properties of these solutions.

Comment on scaling solutions. Consider the special case $j_{\infty}=p+\frac{q}{2}=0$. For this class of solutions (4.4) do not fix the overall size of the system and one can define a scaling limit in which the asymptotically flat region of the metric decouples, resulting in an asymptotically $\mathrm{AdS}_{2} \times S^{3}$ space-time. Since the metric as one approaches any of the finite horizons is also $\mathrm{AdS}_{2} \times S^{3}$, these solutions can be seen as interpolations between different $\mathrm{AdS}_{2} \times S^{3}$ regions. These are usually referred to as scaling solutions [34].

We will now show that, within the choice (4.3), any scaling solution will violate the requirement (4.7a) close to the smooth center. Consider the function $\mathrm{H}_{2} \mathrm{H}=\mathrm{H}_{2} \mathrm{H}_{1}+$ $H_{3} H_{4}$ which must be necessarily positive in order for (4.7a) to be satisfied everywhere. Close to $r=0$ we have

$$
H_{2} H=\frac{q p\left(\left|z_{1}\right|+\left|z_{3}\right|\right)-\mu_{1}\left|z_{3}\right|-\mu_{3}\left|z_{1}\right|-\left|z_{1} z_{3}\right|}{r\left|z_{1} z_{3}\right|}+\mathcal{O}\left(r^{0}\right)>0
$$

which implies

$$
q p>\frac{\mu_{1}\left|z_{3}\right|+\mu_{3}\left|z_{1}\right|+\left|z_{1} z_{3}\right|}{\left|z_{1}\right|+\left|z_{3}\right|}>0 .
$$

However, for a scaling solution we have $q p=-2 p^{2} \leq 0$ and the above is necessarily violated. 


\subsection{Global charges}

The mass, electric charges, and angular momenta of the twin black hole solution are given by the general expressions (3.10), (3.11), (3.12) and read, in this particular case,

$$
\begin{aligned}
& M=8\left(n+p^{2}+2(\mu+p q)\right), \quad Q=8\left(n+p^{2}\right), \quad \widetilde{Q}=8 \sqrt{2}(\mu+p q), \\
& J_{\psi}=8\left(2 j+n q+2 \mu p+3 p^{2} q\right), \quad J_{\phi}=0 .
\end{aligned}
$$

We note that for this system $J_{\phi}=0$, which is a consequence of the bubble equations; computing the angular momentum (3.12) gives $J_{\phi}=\left(p+\frac{q}{2}\right)\left(z_{1}+z_{3}\right)$, which vanishes since $z_{1}=-z_{3}$ as shown above. ${ }^{9}$ Solutions with $J_{\phi} \neq 0$ can be obtained by considering nonidentical black holes.

In addition to the asymptotic charges, the solution is characterized by the fluxes (3.9) through the two independent 2-cycles $\mathcal{C}_{12}$ and $\mathcal{C}_{23}$ of the GH base:

$$
f_{12}=f_{23}=q, \quad \tilde{f}_{12}=\widetilde{f}_{23}=\sqrt{2} p .
$$

Recall that (4.10) requires, in particular, $p q>0$ and thus both fluxes must be nonzero for regularity.

\subsection{Parameter space and entropy}

Fixing the asymptotic charges does not completely specify the three-center solution. Indeed, one may solve say for $n, \mu, q$ in terms of $Q, \widetilde{Q}, J_{\psi}$, leaving the local quantities $j, p$ undetermined (although bounded by the regularity constraints). Thus, for a given set of asymptotic charges, there is a two-parameter family of twin black hole systems with the same asymptotics as the single-center solution. It is natural to compare the entropy of the two configurations. To first approximation, the entropy of the twin black hole system is simply given by the sum of the Bekenstein-Hawking entropies of the two black holes, namely,

$$
\begin{aligned}
S_{\text {twin }} & =32 \pi \sqrt{\mu^{2} n-j^{2}} \\
& =\frac{32 \pi}{Q} \sqrt{2\left[8 j p-\frac{J_{\psi}}{2} p+\frac{\widetilde{Q}}{4 \sqrt{2}}\left(4 p^{2}+\frac{1}{4} Q\right)\right]^{2}\left(\frac{Q}{4}-2 p^{2}\right)-Q^{2} j^{2},}
\end{aligned}
$$

where in the second line we have used (4.14) to write the expression in terms of asymptotic charges. For comparison, the entropy of the single-center BMPV black hole with same asymptotic charges reads

$$
S_{\mathrm{BMPV}}=2 \pi \sqrt{\frac{\widetilde{Q}^{2} Q}{2}-J_{\psi}^{2}} .
$$

At this point one can already see that for some choice of the asymptotic charges the twin black hole system can be entropically favored over the BMPV black hole. Indeed,

\footnotetext{
${ }^{9}$ We note that in the case of scaling solutions $2 p+q=0$ and hence $J_{\phi}=0$, even for nonidentical black holes.
} 
consider a maximally-spinning BMPV black hole, i.e.,

$$
\left|J_{\psi}\right|=\frac{1}{\sqrt{2}} \widetilde{Q} \sqrt{Q}: \quad S_{\mathrm{BMPV}}=0 \quad \text { and } \quad S_{\mathrm{twin}}>0,
$$

provided the requirements (4.9) and (4.10) are satisfied.

We now discuss the region of existence of this solution in parameter space. Before discussing this we note the entropy function has the following useful scaling properties

$$
\begin{aligned}
S\left(Q, \lambda \widetilde{Q}, \lambda J_{\psi}, p, \lambda j\right) & =\lambda S\left(Q, \widetilde{Q}, J_{\psi}, p, j\right), \\
S\left(\Lambda^{2} Q, \widetilde{Q}, \Lambda J_{\psi}, \Lambda p, \Lambda j\right) & =\Lambda S\left(Q, \widetilde{Q}, J_{\psi}, p, j\right),
\end{aligned}
$$

which can be used to write

$$
S\left(Q, \widetilde{Q}, J_{\psi}, p, j\right)=\widetilde{Q} \sqrt{Q} S\left(1,1, \frac{J_{\psi}}{\widetilde{Q} \sqrt{Q}}, \frac{p}{\sqrt{Q}}, \frac{j}{\widetilde{Q} \sqrt{Q}}\right) .
$$

Thus, it is convenient to define

$$
x=\frac{\sqrt{2} J_{\psi}}{\widetilde{Q} \sqrt{Q}}, \quad \alpha=\frac{2 p}{\sqrt{Q}}, \quad \beta=\frac{8 \sqrt{2} j}{\widetilde{Q} \sqrt{Q}},
$$

in terms of which

$$
\frac{\sqrt{2} S_{\text {twin }}}{\widetilde{Q} \sqrt{Q}} \equiv \sigma(\alpha, \beta ; x)=4 \pi \sqrt{\frac{1}{8}\left(1-2 \alpha^{2}\right)(4 \alpha(\alpha+2 \beta-x)+1)^{2}-\beta^{2}}
$$

and

$$
\frac{\sqrt{2} S_{\mathrm{BMPV}}}{\widetilde{Q} \sqrt{Q}}=2 \pi \sqrt{1-x^{2}} .
$$

We note $x=1$ corresponds to the CCB for the single-center.

The five-dimensional parameter space of the twin black solution is spanned by the coordinates $(Q, \widetilde{Q}, x, \alpha, \beta)$. To truly establish the existence and regularity of this solution, one must study whether there exists a region in this space where all the regularity conditions close to the centers (4.6), (4.9), and (4.10) are satisfied. As discussed in subsection 4.2 this is enough to also ensure global regularity in this class of solutions, at least within our numerical analysis of $g^{t t}$. It is easy to see that there exists a five-dimensional region in parameter space where all these conditions are satisfied and, in fact, it is infinite in extent. A more interesting question, however, is whether this is the case if the asymptotic charges $Q, \widetilde{Q}, J_{\psi}$ are fixed, i.e., the two-dimensional slices of this space spanned by the parameters $\alpha, \beta$. The situation is slightly different in the cases $x<1$ and $x \geq 1$, i.e., above and below the CCB of the single-center solution. The schematic situation is shown in figure 3. For $x_{\min }<x<1$ with $x_{\min }=7 \sqrt{2} / 5 \sqrt{5}$ the single-center and twin black solutions coexist. As shown in the left plot, the region where the twin black hole is regular is bounded on one side by the curve $a=0$, where the separation between the black holes goes to zero, and on the other side by the CCB for each individual black hole. For $1 \leq x<x_{\max }$ with $x_{\max }=3 / 2 \sqrt{2}$ (right plot), the curve $g_{\psi \psi}=0$ closes on itself and entirely bounds the region 
$\beta$

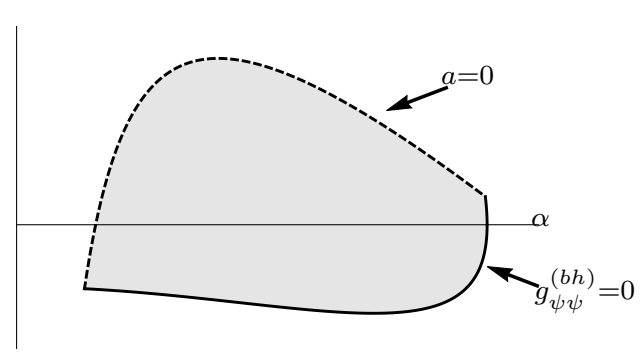

$\beta$

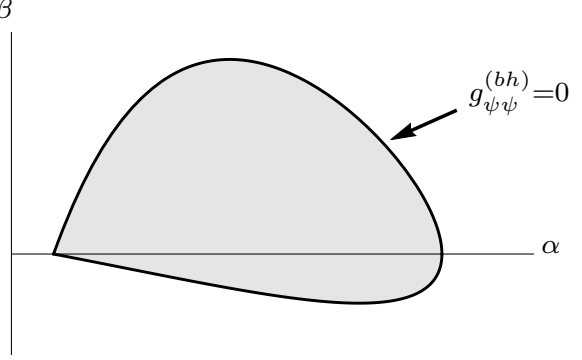

Figure 3. Region in $\alpha, \beta$ plane where the twin black hole solution is regular. For $x_{\min }<x<1$ (left figure) the single-center and twin black solutions coexist. For $1 \leq x<x_{\max }$ (right figure) the single-center solution violates the CCB but the twin black hole with the same asymptotic charges is regular.

of existence. For $x=x_{\min }$ and $x=x_{\max }$ the regions in figure 3 shrink to a point. Although this figure suggests that the other regularity conditions, such as $g_{r r}>0$ or $g_{\psi \psi}>0$ close to the smooth center are unimportant, this is not the case; these are responsible for excluding other regions of parameter space which are not shown in the figure.

We now discuss the entropy of the twin black hole system. For any value of the parameters $\alpha, \beta$ belonging to the region shown in figure 3 there exists a twin black hole solution with the same asymptotic charges as the single-center solution. Although the asymptotic charges are fixed, the entropy (4.16) of the twin black hole system depends on the values of $j, p$. To compare with the single-center entropy we may maximize $S_{\text {twin }}$ with respect to these quantities. Performing this maximization, making sure that the location of the maximum belongs to the regions in figure 3, one obtains the behavior displayed in the left plot in figure 4. For certain values of the the variable $x$, the maximum of the entropy is not an extremum, but in fact lies on the boundary of the allowed region; this is responsible for the kink in $S_{\mathrm{twin}}^{\max }$ in figure 4 .

As shown in the right plot in figure 4 , in $\left(Q, \widetilde{Q}, J_{\psi}\right)$ space there is a narrow region where the twin black holes and the BMPV solution coexist. Namely, where the narrow dark strip overlaps with the interior of the gray region. The parabola represents the CCB for the single-center solution, where its entropy vanishes. In a small neighborhood to the left of the CCB both solutions exist and the twin black hole system has more entropy.

Of course, there is a vast number of multicenter solutions with the same asymptotic charges and some of these may be entropically favored over the twin back hole configuration discussed above. For instance, following the discussion in $[12,33]$ one should expect that, for a given set of asymptotic charges and fixed number of centers, the configuration with just one black center is in general entropically favored over the ones with more black centers. In the present case, this means that the solution with one black hole and two smooth centers (see [13] for a study of this solution) should carry more entropy than the twin black hole system presented in this paper. A numerical analysis shows that this is indeed the case: the solution of [13] is entropically favored over the bound state presented in this paper, in the region of parameter space shared by the two solutions. In principle, a full analysis of the ensemble defined by fixing the asymptotic charges would require one to 

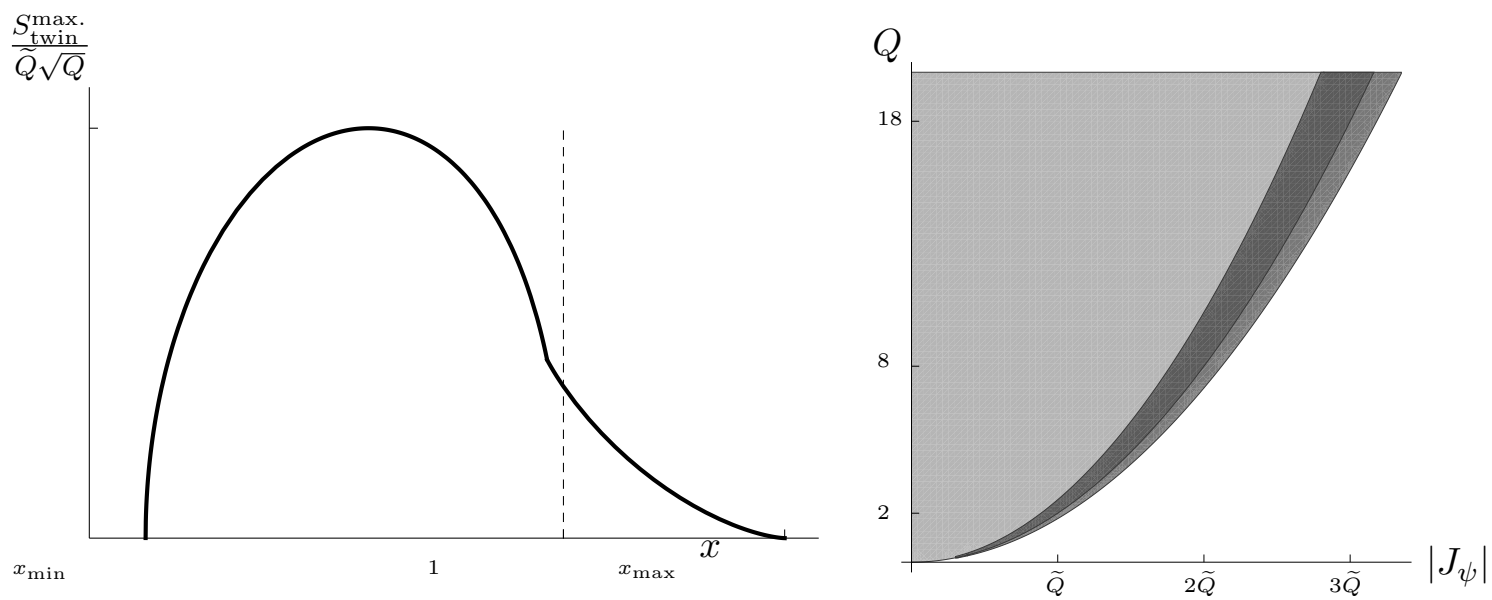

Figure 4. Left plot: maximum of the entropy as a function of $J_{\psi}$, for fixed values of $Q, \widetilde{Q}$. Here $x_{\min }=7 \sqrt{2} / 5 \sqrt{5}$ and $x_{\max }=3 / 2 \sqrt{2}$. The dashed vertical line represents the maximum value of the spin for the BMPV black hole. Right plot: region where the twin black hole solution exists (dark gray) in the $\left(J_{\psi}, Q\right)$ plane, for a fixed value of $\widetilde{Q}$. In the light gray region only the BMPV black hole exists. In the darkest region in the middle the two solutions coexist.

consider even more exotic configurations with arbitrary number of black rings, black holes, black lenses and smooth centers. This is beyond the aim of the present paper.

\section{Summary and discussion}

We have shown the existence of a large class of regular, BPS black hole bound state solutions in asymptotically $\mathbb{R}^{1,4}$ spacetime. These were obtained by dimensional reduction of sixdimensional configurations of strings in minimal $6 \mathrm{~d}(1,0)$ supergravity which admit an uplift to F-theory on an elliptically fibered Calabi-Yau threefold $X$ with base $\mathbb{P}^{2}$. Other threefolds $X$ may also be considered, as long as the low-energy theory can be truncated to minimal $6 \mathrm{~d}$ supergravity with frozen hypermultiplets. Presumably, these black hole bound states still exist in theories with additional supersymmetry, such as toroidal compactifications of M-theory or type IIB theory.

The particular multicenter configurations we have discussed consist of bound states of black holes with $S^{3}$ horizons and smooth, horizonless, centers. We have shown that there is a finite region in parameter space where these configurations coexist with the single-center BMPV black hole with the same asymptotic charges, and a region where the single-center solution would violate the $\mathrm{CCB}$, but the multicenter is regular.

Although a detailed analysis of the regularity constraints imposed on parameter space was carried out only in the three-center case (two black holes and a smooth center), one would expect similar results to hold in the case of an arbitrary number of centers. In particular, one may consider adding $N$ pairs of smooth centers with $m_{a}=-1$ and black holes with $m_{b}=1$ without changing the $\mathbb{R}^{1,4}$ asymptotics.

Clearly, our results ask for a microscopic description. In F-theory, this might be possible using the recent results presented in [9], though we should stress that the bound 
states described here are not the ones described in [9]. The latter are described by states with an entropy that is linear in the charges, and correspond to bound states of small black holes (with Taub-NUT asymptotics), while the bound states described in this paper can consist of large black holes. Moreover, we have additional fluxes that support the bound states which are absent in [9]. Presumably, the microscopics is easier to analyze in toroidal compactifications when the CFT has $(4,4)$ supersymmetry rather than $(0,4)$.

We have also discussed a set of $\operatorname{Sp}(6, \mathbb{R})$ transformations acting nontrivially on the solutions which, nonetheless, as a consequence of preserving the symplectic product, preserve the bubble equations. Subsets of these transformations have been considered in the literature before, including the generalized spectral flow transformations discussed and exploited in [26]. It would be interesting to study the consequence of the full set of $\operatorname{Sp}(6, \mathbb{R})$ transformations [28].

Finally, we comment on the possibility of an ambipolar Taub-NUT base space, in which case the asymptotics in five dimensions would be $\mathbb{R}^{1,3} \times S^{1}$. This is interesting as the $S^{1}$ in the Taub-NUT geometry allows the further dimensional reduction down to four dimensions. The relation among various parameters that ensure the correct asymptotics of the six-dimensional solution are discussed in appendix A.2. Although the setting is very similar to the asymptotically $\mathbb{R}^{1,4}$ case, to truly establish the existence and regularity of these solutions one must repeat the analysis performed above, which is left for future work.

\section{Acknowledgments}

We thank Nikolay Bobev, Nava Gaddam and Sameer Murthy for comments and feedback. The authors are supported by the Netherlands Organization for Scientific Research (NWO) under the VICI Grant 680-47-603. This work is part of the D-ITP consortium, a program of the NWO that is funded by the Dutch Ministry of Education, Culture and Science (OCW).

\section{A $6 \mathrm{~d}$ solution and reduction to $5 \mathrm{~d}$}

In this appendix, we provide some details of the six- and five-dimensional solutions discussed in the main text.

\section{A.1 The 1-forms $\chi, \beta, \omega$}

To fully specify the metric (3.2) one must determine the 1 -forms $\boldsymbol{\chi}, \boldsymbol{\beta}, \boldsymbol{\omega}$ from equations (2.8), (2.12) and (2.13), respectively. For simplicity, we restrict ourselves to axisymmetric configurations, with all $N$ centers lying on a single line, which we take to be the $z$-axis, located at $\theta=(0, \pi)$. In this case it is easy to solve these equations, obtaining

$$
\chi=\sum_{a=1}^{N} m_{a} \frac{r \cos (\theta)-z_{a}}{r_{a}} \mathrm{~d} \phi, \quad \boldsymbol{\beta}=-\sum_{a=1}^{N} q_{a} \frac{r \cos (\theta)-z_{a}}{r_{a}} \mathrm{~d} \phi,
$$

and

$$
\boldsymbol{\omega}=\frac{1}{2} \sum_{\substack{\{a, b\}=1 \\ a \neq b}}^{N}\left\langle\Gamma_{a}, \Gamma_{b}\right\rangle \frac{\left(z_{a}-z_{b}\right)^{2}-\left(r_{a}-r_{b}\right)^{2}}{2\left(z_{a}-z_{b}\right) r_{a} r_{b}} \mathrm{~d} \phi+\sum_{a=1}^{N}\left\langle\Gamma_{\infty}, \Gamma_{a}\right\rangle \frac{r \cos (\theta)-z_{a}}{r_{a}} \mathrm{~d} \phi
$$


where $r_{a} \equiv\left|\vec{x}-\vec{x}_{a}\right|=\sqrt{r^{2}+z_{a}^{2}-2 r z_{a} \cos \theta}$. Of course, these 1-forms are defined up to gauge transformations; we have chosen a gauge in (A.2) such that asymptotically $\boldsymbol{\omega} \rightarrow$ $\left\langle\Gamma_{\infty}, \Gamma_{\mathrm{T}}\right\rangle \cos \theta \mathrm{d} \phi$, which vanishes when (2.23) is imposed.

We note that evaluating (A.2) on the (positive) $z$-axis gives

$$
\left.\boldsymbol{\omega}\right|_{\theta=0}=\sum_{a=1}^{N} \operatorname{sign}\left(r-z_{a}\right)\left(\left\langle\Gamma_{\infty}, \Gamma_{a}\right\rangle-\sum_{b \neq a} \frac{\left\langle\Gamma_{a}, \Gamma_{b}\right\rangle}{\left|z_{a}-z_{b}\right|}\right) \mathrm{d} \phi .
$$

Since the function $\operatorname{sign}\left(r-z_{a}\right)$ is discontinuous across the centers, the bubble equations (2.22) ensure that $\boldsymbol{\omega}$ is regular (in fact, vanishing) along the axis. ${ }^{10}$

\section{A.2 Asymptotics}

Requiring that asymptotically $(r \rightarrow \infty)$ the functions $H, \mathcal{F} \rightarrow 1$ and the 1 -forms $\omega, \beta \rightarrow 0$, the metric (2.3) asymptotes to $\mathbb{R}^{1} \times S_{u}^{1} \times X$, where $X$ is the asymptotics of the GibbonsHawking base. ${ }^{11}$ Setting $m_{\infty}=0$ and $m_{\mathrm{T}}=\sum_{a} m_{a}=1$ one has $X=\mathbb{R}^{4}$, while setting $m_{\infty}=1$ gives $X=\mathbb{R}^{3} \times S^{1}$.

In each case, this requirement leads to different relations among the parameters describing the solution. Setting $m_{\infty}=0$ and $m_{\mathrm{T}}=1$ and requiring that $H, \mathcal{F} \rightarrow 1$ and that $\omega, \beta \rightarrow 0$ one finds

$$
\mu_{\infty}=n_{\infty}=1, \quad p_{\infty}=q_{\infty}=0
$$

Then the 1-forms are given asymptotically by

$$
\beta=q_{\mathrm{T}} \mathrm{d} \psi+\mathcal{O}\left(r^{-1}\right), \quad \omega=-\left\langle\Gamma_{\infty}, \Gamma_{\mathrm{T}}\right\rangle \mathrm{d} \psi+\mathcal{O}\left(r^{-1}\right) .
$$

Imposing the bubble equation (2.23) implies $\omega$ identically vanishes at infinity in this gauge. The asymptotic form of $\beta$ can be absorbed by the simple coordinate redefinition $\mathrm{d} u \rightarrow$ $\mathrm{d} u-q_{\mathrm{T}} \mathrm{d} \psi$. Note that, if the coordiante $u$ is periodic, then this is a change of coordinates on a torus and for it to be well-defined one must require

$$
\frac{\ell q_{\mathrm{T}}}{4 \pi} \in \mathbb{Z} .
$$

When the $u$-direction is compacified, this is a necessary condition for the space to be asymptotically $\mathbb{R}^{1,4} \times S_{u}^{1}$ with no conical defect. Imposing the constant term in $\omega$ to vanish we obtain

$$
\Gamma_{\infty}=\left(1,0,0,0,1,-p_{\mathrm{T}}-\frac{q_{\mathrm{T}}}{2}\right)
$$

where $x_{\mathrm{T}} \equiv \sum_{a} x_{a}$.

In the case of Taub-NUT, setting $m_{\infty}=1$ and imposing that $H, \mathcal{F} \rightarrow 1$ requires

$$
\mu_{\infty}+p_{\infty} q_{\infty}=1, \quad n_{\infty}+p_{\infty}^{2}=1
$$

\footnotetext{
${ }^{10}$ The expression on the negative $z$-axis is obtained by sending $r \rightarrow-r$ in this expression, and the same conclusion follows.

${ }^{11}$ One can also relax the condition to $H^{2} \mathcal{F} \rightarrow 1$ thus allowing for $H \rightarrow \mu_{\infty}$. This will rescale the radius of the asymptotic $S_{u}^{1}$. Here we fix $\mu_{\infty}=1$.
} 
In this case, the 1-forms are given asymptotically by

$$
\begin{aligned}
& \beta=q_{\infty} \mathrm{d} \psi+\left(m_{\mathrm{T}} q_{\infty}-q_{\mathrm{T}}\right) \cos \theta \mathrm{d} \phi+\mathcal{O}\left(r^{-1}\right), \\
& \omega=J_{\psi \infty} \mathrm{d} \psi+\left(m_{\mathrm{T}} J_{\psi \infty}+\left\langle\Gamma_{\infty}, \Gamma_{\mathrm{T}}\right\rangle\right) \cos \theta \mathrm{d} \phi+\mathcal{O}\left(r^{-1}\right),
\end{aligned}
$$

where $J_{\psi \infty}$ is as in (2.26). Thus, if the bubble equation (2.23) is imposed one must set

$$
m_{\mathrm{T}} q_{\infty}=q_{\mathrm{T}}, \quad m_{\mathrm{T}} J_{\psi \infty}=0 .
$$

Assuming $m_{\mathrm{T}} \neq 0$ the vector $\Gamma_{\infty}$ therefore reads

$$
\Gamma_{\infty}=\left(1-\frac{p_{\infty} q_{\mathrm{T}}}{m_{\mathrm{T}}}, 1, \frac{q_{\mathrm{T}}}{m_{\mathrm{T}}}, p_{\infty}, 1-p_{\infty}^{2},-p_{\infty}-\frac{q_{\mathrm{T}}\left(1-p_{\infty}^{2}\right)}{2 m_{\mathrm{T}}}\right) .
$$

The parameter $p_{\infty}$ is fixed in terms of $\Gamma_{\mathrm{T}}$ from $\left\langle\Gamma_{\infty}, \Gamma_{\mathrm{T}}\right\rangle=0$.

\section{A.3 Reduction to $5 \mathrm{~d}$}

The generic metric Ansatz for the reduction along a spacelike direction $u$ is

$$
\begin{aligned}
\mathrm{d} s_{6}^{2} & =\mathrm{e}^{2 \varphi}(\mathrm{d} u+A)^{2}+\mathrm{e}^{2 \alpha \varphi} \mathrm{d} s_{5}^{2}, \\
\widehat{G} & =G+\frac{1}{2} \mathrm{~d} \widetilde{A} \wedge(\mathrm{d} u+A),
\end{aligned}
$$

where $\alpha$ is an arbitrary constant. Upon dimensional reduction along $u \mathrm{~d} s_{5}^{2}$ becomes the five-dimensional metric, $\varphi$ becomes the dilaton field, $A$ the graviphoton, $G$ a 3 -form field in five dimensions and $\widetilde{A}$ another vector multiplet. The 6d Einstein-Hilbert Lagrangian, in terms of the $5 \mathrm{~d}$ fields reads

$$
\sqrt{-g^{(6)}} R^{(6)}=\sqrt{g^{(5)}} \mathrm{e}^{(3 \alpha+1) \varphi}\left(R^{(5)}-2 \mathbf{c}(\partial \varphi)^{2}-\frac{1}{4} \mathrm{e}^{-2(\alpha-1) \varphi} F^{2}\right),
$$

where $F=\mathrm{d} A$ and $\mathbf{c}=\left(6 \alpha^{2}+3 \alpha+1\right)$. If we demand the $5 \mathrm{~d}$ Lagrangian to contain a canonical Einstein Hilbert term (Einstein frame) we must choose $\alpha=-1 / 3$.

We now apply this generic reduction to the solutions at hand. We assume the solution (2.3) is $u$-independent and reduce along this direction. The first step is to bring the metric into the form (A.11), namely:

$$
\mathrm{d} s_{6}^{2}=H^{-1} \mathcal{F}\left(\mathrm{d} u+\beta-\mathcal{F}^{-1}(\mathrm{~d} v+\omega)\right)^{2}-H^{-1} \mathcal{F}^{-1}(\mathrm{~d} v+\omega)^{2}+H \mathrm{~d} s_{4}^{2} .
$$

Comparing this to (A.11), we identify the dilaton

$$
\mathrm{e}^{2 \varphi}=H^{-1} \mathcal{F}
$$

and the five-dimensional metric in Einstein frame reads:

$$
\mathrm{d} s_{5}^{2}=-\frac{1}{\left(H^{2} \mathcal{F}\right)^{2 / 3}}(\mathrm{~d} t+w)^{2}+\left(H^{2} \mathcal{F}\right)^{1 / 3} \mathrm{~d} s_{4}^{2},
$$

where we renamed $\mathrm{d} v=\mathrm{d} t$ and $t$ is a time-like direction in $5 \mathrm{~d}$. The graviphoton is given by

$$
A=\beta-\mathcal{F}^{-1}(\mathrm{~d} t+\omega) .
$$


Now we turn to the reduction of the three-form field. In six dimensions, it is given by (see [17])

$$
\widehat{G}=\frac{1}{2} *_{4} \mathrm{~d} H-\frac{1}{2} e^{+} \wedge(\mathrm{d} \omega)^{-}+\frac{1}{2} H^{-1} e^{-} \wedge \mathrm{d} \beta-\frac{1}{2} e^{+} \wedge e^{-} \wedge H^{-1} \mathrm{~d} H,
$$

where

$$
e^{+}=H^{-1}(\mathrm{~d} u+\beta), \quad e^{-}=\mathrm{d} v+\omega-\frac{\mathcal{F}}{2}(\mathrm{~d} u+\beta) .
$$

In order to bring this into the form form (A.11) we perform some simple manipulations to write

$\widehat{G}=-\frac{1}{2}\left(\mathrm{~d}\left(H^{-1}(\mathrm{~d} v+\omega)\right)-\mathcal{G}^{+}\right) \wedge(\mathrm{d} u+A)+\frac{1}{2} *_{4} \mathrm{~d} H+\frac{1}{2} H^{-1}(\mathrm{~d} v+\omega) \wedge\left(\frac{1}{2} \mathrm{~d} \beta-\mathcal{F}^{-1}(\mathrm{~d} \omega)^{-}\right)$

where we used $\mathrm{d} \omega^{-}=\frac{1}{2}\left(\mathrm{~d} \omega-*_{4} \mathrm{~d} \omega\right)$ and defined

$$
\mathcal{G}^{+}=H^{-1}\left((\mathrm{~d} \omega)^{+}-\frac{1}{2} \mathcal{F} \mathrm{d} \beta\right)
$$

For this class of solutions

$$
\mathcal{G}^{+}=\partial_{i}\left(H_{2}^{-1} H_{4}\right) \mathrm{d} x^{i} \wedge \sigma-\frac{1}{2} \epsilon_{i j k} \partial_{k}\left(H_{2}^{-1} H_{4}\right) H_{2} \mathrm{~d} x^{i} \wedge \mathrm{d} x^{j},
$$

which can be written as

$$
\mathcal{G}^{+}=\mathrm{d}\left(H_{2}^{-1} H_{4} \sigma\right)-*_{3} \mathrm{~d} H_{4}=\mathrm{d}\left(H_{2}^{-1} H_{4} \sigma+\gamma\right)
$$

where in the second equality we introduced the 1-form $\gamma$ satisfying

$$
*_{3} \mathrm{~d} \gamma=-\mathrm{d} H_{4} .
$$

Combining this with (A.19) we have

$$
\begin{aligned}
\widehat{G}= & \frac{1}{2} \mathrm{~d}\left[-H^{-1}(\mathrm{~d} v+\omega)+H_{2}^{-1} H_{4} \sigma+\gamma\right] \wedge(\mathrm{d} u+A) \\
& +\frac{1}{2} *_{4} \mathrm{~d} H+\frac{1}{2} H^{-1}(\mathrm{~d} v+\omega) \wedge\left(\frac{1}{2} \mathrm{~d} \beta-\mathcal{F}^{-1}(\mathrm{~d} \omega)^{-}\right) .
\end{aligned}
$$

We may now compare this expression to (A.11) and identify the five-dimensional fields

$$
\begin{aligned}
\widetilde{A} & =-H^{-1}(\mathrm{~d} t+\omega)+H_{2}^{-1} H_{4} \sigma+\gamma \\
G & =\frac{1}{2} *_{4} \mathrm{~d} H+\frac{1}{2} H^{-1}(\mathrm{~d} t+\omega) \wedge\left(\frac{1}{2} \mathrm{~d} \beta-\mathcal{F}^{-1}(\mathrm{~d} \omega)^{-}\right),
\end{aligned}
$$

where $\mathrm{d} t=\mathrm{d} v$. Due to the self-duality of the 3 -form $\widehat{G}$ in six dimensions, the 3 -form $G$ in $5 \mathrm{~d}$ is related to ${ }_{5} \mathrm{~d} \widetilde{A}$ and is not an independent field. The field strengths of the two vector fields read:

$$
\begin{aligned}
& F=\mathrm{d} A=\mathcal{F}^{-2} \partial_{i} \mathcal{F} \mathrm{d} x^{i} \wedge(\mathrm{d} t+\omega)+\mathrm{d} \beta-\mathcal{F}^{-1} \mathrm{~d} \omega \\
& \widetilde{F}=\mathrm{d} \widetilde{A}=-\mathrm{d}\left(H^{-1}(\mathrm{~d} t+\omega)\right)+\mathcal{G}^{+}
\end{aligned}
$$




\section{B Five-dimensional metrics}

Here we provide some details of the five-dimensional metrics studied in this paper. We discuss their asymptotics, their behavior close to the GH centers, and compute their physical properties (mass, electric charges, and magnetic fluxes) for solutions with an arbitrary number of centers. We also provide some details of the regularity constraints.

\section{B.1 Asymptotics}

Setting $\Gamma_{\infty}$ to (A.7), and $m=1$, and reducing along the $u$-direction leads to a fivedimensional solution asymptotic to $\mathbb{R} \times \mathbb{R}^{4}$ while setting $\Gamma_{\infty}$ to (A.10) (for $m \neq 0$ ) leads to $\mathbb{R} \times \mathbb{R}^{3} \times S^{1}$ asymptotics.

Other interesting boundary conditions that one may consider in five dimensions are $\mathrm{AdS}_{2} \times S^{3}$ or $\mathrm{AdS}_{3} \times S^{2}$, obtained by setting

$$
\begin{array}{lll}
\mathrm{AdS}_{2} \times S^{3}: & \Gamma_{\infty}=0, & m_{\mathrm{T}}=1, \\
\mathrm{AdS}_{3} \times S^{2}: & \Gamma_{\infty}=0, & m_{\mathrm{T}}=0 .
\end{array}
$$

\section{B.2 Near a center}

Close to a center $\vec{x}_{a}$ the metric functions behave like:

$$
\begin{aligned}
H & =H_{a}+\frac{\widetilde{Q}_{a}}{4 \sqrt{2} m_{a} r_{a}}+\mathcal{O}\left(r_{a}\right), & \mathcal{F} & =\mathcal{F}_{a}+\frac{Q_{a}}{4 m_{a} r_{a}}+\mathcal{O}\left(r_{a}\right), \\
\omega_{\psi} & =\omega_{\psi}^{a}+\frac{J_{a}^{\psi}}{8 m_{a}^{2} r_{a}}+\mathcal{O}\left(r_{a}\right), & \boldsymbol{\omega}_{i} & =\mathcal{O}\left(r_{a}\right),
\end{aligned}
$$

where $\widetilde{Q}_{a}, Q_{a}, J_{a}^{\psi}$ are given in $(2.26)$ and $H_{a}, \mathcal{F}_{a}, \omega_{\psi}^{a}$ are constants which depend on the charges of all the centers, as well as their locations. The fact that $\boldsymbol{\omega}$ vanishes to leading order is a consequence of the bubble equations (see e.g. A.3). For a generic $\Gamma_{a}$ the $r_{a}^{-1}$ terms above are dominant. However, if the coefficients of these terms vanish, the leading behavior is controlled by the constant parts; the metric behaves quite differently in these two cases. As we discuss now, in the former case the near-center metric coincides with (near-horizon) metric of a black hole with a finite-size horizon of topology $S^{3} / \mathbb{Z}_{\left|m_{a}\right|}$. In the latter case there is no horizon and the near-center metric is $\mathbb{R}^{1,4} / \mathbb{Z}_{\left|m_{a}\right|}$.

Centers with $\boldsymbol{S}^{\mathbf{3}}$ horizons. Assuming $\left(\widetilde{Q}_{a}, Q_{a}, J_{a}^{\psi}\right) \neq 0$ the $1 / r_{a}$ parts in (B.4) are dominant and by a simple change of coordinates the metric near the center reads

$$
\begin{aligned}
\mathrm{d} s_{5}^{2} \simeq & -\frac{r_{a}^{2} \mathrm{~d} v^{2}}{\alpha_{a} m_{a} \lambda_{a}} \pm \frac{2 \alpha_{a} \mathrm{~d} v \mathrm{~d} r_{a}}{\sqrt{\alpha_{a}^{2} \lambda_{a}}}+\lambda_{a}\left(\mathrm{~d} \psi^{\prime}+\chi_{\phi}^{(0)} \mathrm{d} \phi-\frac{r_{a} \sqrt{\alpha_{a}-m_{a} \lambda_{a}}}{\lambda_{a} \alpha_{a} \sqrt{m_{a}}} \mathrm{~d} v\right)^{2} \\
& +\alpha_{a} m_{a}\left(\mathrm{~d} \theta^{2}+\sin ^{2} \theta \mathrm{d} \phi^{2}\right)
\end{aligned}
$$

where

$$
\alpha_{a}=\frac{1}{4 m_{a}}\left(\frac{\widetilde{Q}_{a}^{2} Q_{a}}{2}\right)^{1 / 3}, \quad \lambda_{a} \equiv \frac{\alpha_{a}}{m_{a}}-\frac{\left(J_{a}^{\psi}\right)^{2}}{64 m_{a}^{4} \alpha_{a}^{2}}
$$


The metric (B.5) can be shown to correspond to the near horizon of a BMPV black hole [1], the two signs corresponding to a future or past horizon (see [13] for a discussion). In particular the entropy associated to the center at $r_{a}=0$ is

$$
S_{a}=\frac{\mathcal{A}_{a}}{4 G_{5}}=\frac{1}{\left|m_{a}\right|} 2 \pi \sqrt{\frac{\widetilde{Q}_{a}^{2} Q_{a}}{2}-J_{a}^{2}} .
$$

In case of the single GH center with $m_{a}=1, q_{a}=p_{a}=0, j_{a}=j, \mu_{a}=\mu$ this becomes the entropy of the BMPV black hole $S=16 \pi \sqrt{\mu^{2} n-j^{2}}$.

For the metric to be regular one must impose

$$
\widetilde{Q}_{a}>0, \quad Q_{a}>0, \quad \frac{\widetilde{Q}_{a}^{2} Q_{a}}{2}-\left(J_{a}^{\psi}\right)^{2}>0 .
$$

In the case of a single center BMPV black hole the last condition above is the usual CCB.

Smooth horizonless centers. An interesting class of solutions are those for which

$$
\widetilde{Q}_{a}=Q_{a}=J_{a}^{\psi}=0 .
$$

In this case the functions (B.4) remain finite close to the center. For smooth centers the constant part $\omega_{\psi}^{a}$ is given by

$$
\omega_{\psi}^{a}=\frac{1}{m_{a}}\left(\sum_{b \neq a} \frac{\left\langle\Gamma_{a}, \Gamma_{b}\right\rangle}{r_{a b}}-\left\langle\Gamma_{\infty}, \Gamma_{a}\right\rangle\right),
$$

which vanishes when the bubble equations are imposed. To avoid orbifold singularities in the base we set $m_{a}= \pm 1$. Thus, close to a GH center satisfying (B.9), the metric is simply

$$
\mathrm{d} s_{5}^{2} \simeq-\left(H_{a}^{2} \mathcal{F}_{a}\right)^{-2 / 3} \mathrm{~d} t^{2}+\left(H_{a}^{2} \mathcal{F}_{a}\right)^{1 / 3} \mathrm{~d} s_{ \pm \mathbb{R}^{4}}^{2}
$$

where $\mathrm{d} s_{ \pm \mathbb{R}^{4}}^{2}= \pm \mathrm{d} s_{\mathbb{R}^{4}}^{2}$, where the two signs correspond to the sign of $m_{a}$. One can check that $\operatorname{sign}\left(H_{a}^{2} \mathcal{F}_{a}\right)=\operatorname{sign}\left(m_{a}\right)$, and thus the metric is smooth $\mathbb{R}^{1,4}$. In six dimensions, this uplifts to smooth $\mathbb{R}^{1,5}$.

Finally, close to a center with $m_{a}=0$ the metric looks like the $r \rightarrow 0$ limit of the single black string metric (2.17).

Open Access. This article is distributed under the terms of the Creative Commons Attribution License (CC-BY 4.0), which permits any use, distribution and reproduction in any medium, provided the original author(s) and source are credited.

\section{References}

[1] J.C. Breckenridge, R.C. Myers, A.W. Peet and C. Vafa, D-branes and spinning black holes, Phys. Lett. B 391 (1997) 93 [hep-th/9602065] [INSPIRE].

[2] H. Elvang, R. Emparan, D. Mateos and H.S. Reall, A supersymmetric black ring, Phys. Rev. Lett. 93 (2004) 211302 [hep-th/0407065] [INSPIRE]. 
[3] H.K. Kunduri and J. Lucietti, Supersymmetric Black Holes with Lens-Space Topology, Phys. Rev. Lett. 113 (2014) 211101 [arXiv: 1408.6083] [INSPIRE].

[4] G.W. Gibbons and N.P. Warner, Global structure of five-dimensional fuzzballs, Class. Quant. Grav. 31 (2014) 025016 [arXiv: 1305.0957] [INSPIRE].

[5] J.P. Gauntlett and J.B. Gutowski, Concentric black rings, Phys. Rev. D 71 (2005) 025013 [hep-th/0408010] [INSPIRE].

[6] J.P. Gauntlett and J.B. Gutowski, General concentric black rings, Phys. Rev. D 71 (2005) 045002 [hep-th/0408122] [INSPIRE].

[7] I. Bena and N.P. Warner, Black Holes, Black Rings and Their Microstates, Lect. Notes Phys. 755 (2008) 1 [hep-th/0701216].

[8] C. Vafa, Black holes and Calabi-Yau threefolds, Adv. Theor. Math. Phys. 2 (1998) 207 [hep-th/9711067] [INSPIRE].

[9] B. Haghighat, S. Murthy, C. Vafa and S. Vandoren, F-Theory, Spinning Black Holes and Multi-string Branches, JHEP 01 (2016) 009 [arXiv: 1509.00455] [INSPIRE].

[10] J.P. Gauntlett, R.C. Myers and P.K. Townsend, Black holes of D $=5$ supergravity, Class. Quant. Grav. 16 (1999) 1 [hep-th/9810204] [INSPIRE].

[11] J.P. Gauntlett, J.B. Gutowski, C.M. Hull, S. Pakis and H.S. Reall, All supersymmetric solutions of minimal supergravity in five-dimensions, Class. Quant. Grav. 20 (2003) 4587 [hep-th/0209114] [INSPIRE].

[12] I. Bena, B.D. Chowdhury, J. de Boer, S. El-Showk and M. Shigemori, Moulting black holes, JHEP 03 (2012) 094 [arXiv:1108.0411] [INSPIRE].

[13] H.K. Kunduri and J. Lucietti, Black hole non-uniqueness via spacetime topology in five dimensions, JHEP 10 (2014) 082 [arXiv:1407.8002] [INSPIRE].

[14] C. Vafa, Evidence for F-theory, Nucl. Phys. B 469 (1996) 403 [hep-th/9602022] [InSPIRE].

[15] D.R. Morrison and C. Vafa, Compactifications of F-theory on Calabi-Yau threefolds. 1, Nucl. Phys. B 473 (1996) 74 [hep-th/9602114] [INSPIRE].

[16] D.R. Morrison and C. Vafa, Compactifications of F-theory on Calabi-Yau threefolds. 2., Nucl. Phys. B 476 (1996) 437 [hep-th/9603161] [INSPIRE].

[17] J.B. Gutowski, D. Martelli and H.S. Reall, All supersymmetric solutions of minimal supergravity in six-dimensions, Class. Quant. Grav. 20 (2003) 5049 [hep-th/0306235] [INSPIRE].

[18] I. Bena, D.-E. Diaconescu and B. Florea, Black string entropy and Fourier-Mukai transform, JHEP 04 (2007) 045 [hep-th/0610068] [INSPIRE].

[19] I. Bena, S. Giusto, M. Shigemori and N.P. Warner, Supersymmetric solutions in six dimensions: a linear structure, JHEP 03 (2012) 084 [arXiv:1110.2781] [INSPIRE].

[20] G.W. Gibbons and S.W. Hawking, Gravitational multi-instantons, Phys. Lett. B 78 (1978) 430 [INSPIRE].

[21] I. Bena and N.P. Warner, Bubbling supertubes and foaming black holes, Phys. Rev. D 74 (2006) 066001 [hep-th/0505166] [INSPIRE].

[22] P. Berglund, E.G. Gimon and T.S. Levi, Supergravity microstates for BPS black holes and black rings, JHEP 06 (2006) 007 [hep-th/0505167] [INSPIRE]. 
[23] A. Saxena, G. Potvin, S. Giusto and A.W. Peet, Smooth geometries with four charges in four dimensions, JHEP 04 (2006) 010 [hep-th/0509214] [INSPIRE].

[24] N. Bobev, B.E. Niehoff and N.P. Warner, New supersymmetric bubbles on $A d S_{3} \times S^{3}$, JHEP 10 (2012) 013 [arXiv: 1204.1972] [INSPIRE].

[25] F. Denef, Supergravity flows and D-brane stability, JHEP 08 (2000) 050 [hep-th/0005049] [INSPIRE].

[26] I. Bena, N. Bobev and N.P. Warner, Spectral Flow and the Spectrum of Multi-Center Solutions, Phys. Rev. D 77 (2008) 125025 [arXiv:0803.1203] [INSPIRE].

[27] I. Bena, P. Kraus and N.P. Warner, Black rings in Taub-NUT, Phys. Rev. D 72 (2005) 084019 [hep-th/0504142] [INSPIRE].

[28] M. Crichigno, F. Porri and S. Vandoren, to appear.

[29] A.C. Cadavid, A. Ceresole, R. D'Auria and S. Ferrara, Eleven-dimensional supergravity compactified on Calabi-Yau threefolds, Phys. Lett. B 357 (1995) 76 [hep-th/9506144] [INSPIRE].

[30] H. Looyestijn, E. Plauschinn and S. Vandoren, New potentials from Scherk-Schwarz reductions, JHEP 12 (2010) 016 [arXiv:1008.4286] [INSPIRE].

[31] S. Ferrara and R. Kallosh, Supersymmetry and attractors, Phys. Rev. D 54 (1996) 1514 [hep-th/9602136] [INSPIRE].

[32] S. Ferrara and R. Kallosh, Universality of supersymmetric attractors, Phys. Rev. D 54 (1996) 1525 [hep-th/9603090] [INSPIRE].

[33] J. de Boer, F. Denef, S. El-Showk, I. Messamah and D. Van den Bleeken, Black hole bound states in $A d S_{3} \times S^{2}$, JHEP 11 (2008) 050 [arXiv:0802.2257] [INSPIRE].

[34] I. Bena, C.-W. Wang and N.P. Warner, Mergers and typical black hole microstates, JHEP 11 (2006) 042 [hep-th/0608217] [INSPIRE].

[35] S.W. Hawking, The Chronology protection conjecture, Phys. Rev. D 46 (1992) 603 [InSPIRE].

[36] S.W. Hawking and G.F.R. Ellis, The Large Scale Structure of Space-Time, Cambridge Monographs on Mathematical Physics, Cambridge University Press, Cambridge U.K. (2011). 